\title{
Effect of surface tension and depolarization field on ferroelectric nanomaterials properties
}

\author{
M.D.Glinchuk, A.N.Morozovskaya \\ Institute for Problems of Materials Science, NASc of Ukraine, \\ Krjijanovskogo 3, 03680 Kiev-142, Ukraine
}

\begin{abstract}
The theory of size effects of the properties of nanocrystalline ferroelectric ceramic or nanoparticle powder allowing for surface tension and depolarization field is proposed. Surface tension was included into free energy functional and surface energy was expressed via surface tension coefficient. The latter was shown to be dependent on temperature due to its relation to dielectric permittivity of the nanoparticles. The depolarization field effect was calculated in the model taking into account the space charge layer on the surface, this space-charge being able to compensate depolarization field in the bulk material.

Euler-Lagrange Equation for inhomogeneous polarization of nanomaterial with boundary condition where extrapolation length was shown to be temperature dependent quantity was solved analytically both in paraelectric and ferroelectric phase of size driven phase transition. This phase transition critical temperature dependence on the particle size was calculated. Temperature and size dependence of nanomaterials polarization and dielectric susceptibility was obtained. The possibility to calculate these and other properties by minimization of conventional free energy in the form of different power polarization series, but with the coefficients which depend on particles size, temperature, contribution of depolarization field and surface tension coefficient
\end{abstract}


was demonstrated. These latter effects were shown to influence essentially the nanomaterial properties. The comparison with available experimental data is performed.

\section{INTRODUCTION}

The characteristic feature of ferroelectric nanomaterials (e.g. the ceramics with nanosize grains or powder built by nanosize particles) is known to be inhomogeneous polarization, dielectric susceptibility and other properties (see e.g. ref. [1], [2], [3] and ref. therein). The properties inhomogeneity is related to the strong influence of the surface, where the properties differ from those in the bulk. Because of this surface and correlation energy (polarization gradient $\nabla P$ ) have to be included when calculating the nanomaterials properties. In phenomenological approach for ferroelectrics with perofskite structure the additional terms to free energy density have the form $\delta(\nabla P)^{2} / 2$ and $\delta P_{s}^{2} / 2 \lambda$, where $P_{s}$ is polarization on the surface, $\lambda$ and $\delta$ are material constants. The variation of free energy functional is usually performed to obtain equilibrium polarization. This leads to Euler-Lagrange equation for the polarization with boundary conditions, which include the extrapolation length $\lambda$ (see e.g. ref. [4]) as combined characteristic of surface energy and polarization gradient. Moreover, the extrapolation length value reflects the degree of the polarization inhomogeneity. It is because the boundary condition has the form of tangent line to the polarization on the sur-

face (e.g. for spherical particle with radius $R$ the boundary condition is $\left(\lambda \frac{d P}{d r}+P\right)_{r=R}=0$ ), so that the smaller homogeneity of polarization the larger the extrapolation length and vise versa. Therefore it is important to calculate $\lambda$ value rather than to consider it as phenomenological parameter. While parameter $\delta$ can be estimated via the material correlation radius nothing is known about $\lambda$ value. On the other hand calculation of $\lambda$ in Ising model includes mainly the change of the average surface coordinate number (see e.g. ref. [4], [5] and ref. therein) that seems to be oversimplification for $\lambda$ value estimation.

Another problem is related to contribution of depolarization field effect. The influence 
of this field that is able to destroy spontaneous polarization even in bulk materials can be decreased by such internal factors as free carriers and domain structure and it can be completely compensated under short circuit conditions. It was shown that inhomogeneous polarization in the ferroelectric films makes impossible to compensate the depolarization field in short circuited films made from single domain ferroelectric, that is perfect insulator [6]. However in the majority of works the influence of depolarization field in nanomaterials has not been considered yet. In ref. [7] the influence of depolarization field was considered in the model of the multidomain ferroelectric of finite size and space-charge layers on the surfaces. However the surface energy of the particles was not taken into account, which is known to play the decisive role in the physics of nanomaterials. The important role of the surface tension was demonstrated in [8], where observed size dependence of the phonon modes frequencies in nanocrystalline $\mathrm{PbTiO}_{3}$ was shown to be attributed to the surface tension.

In the present work we propose the model for consideration of depolarization field effect. We present the surface energy via surface tension that made it possible to express the extrapolation length via surface tension coefficient. This coefficient has been shown to be dependent on temperature $T$, that leads to extrapolation length dependence on $T$ and to appearance of some additional temperature dependence of the properties. The ferroelectric nanoparticles are supposed to be single domain (e.g. polarization $P \equiv P_{z}$ ) with spacecharge layer on the surface. The solution of Euler-Lagrange equation for polarization both in paraelectric and ferroelectric phases has shown the possibility to obtain the nanomaterials properties from free energy expression in the form of power series of polarization like that in the bulk, but with renormalized coefficients. These coefficients depend on temperature and the particles sizes. Although there is a distribution of these sizes in nanomaterials, so that the properties have to be averages with the sizes distribution function, we consider only the average radius $R$ of the particles. In many experimental works (see e.g. ref. [1], [2]) the properties of nanomaterials were measured as a function of a particle average size. The average size can be determined from integrated width of diffraction peak using Scherrer's 
formula or with the help of TEM method. Both results have been shown to be in a fairly good agreement with each other [1].

\section{SURFACE ENERGY AND SURFACE TENSION}

\section{A. Surface energy}

Surface energy is known to be the energy of surface tension on the boundary between two media (see e.g. ref. [9]). It was included into thermodynamic potential written for ferroelectric nanomaterial in ref. [10] both as a surface energy and as hydrostatic pressure, acting on nanoparticles. However these terms related to surface tension were not expressed via electric polarization (order parameter of ferroelectrics), so that it appeared impossible to incorporate them directly into Landau free energy that has the form of power series of

polarization. This incorporation seems to be important to pour light on physical meaning of some parameters of thermodynamic theory approach to nanomaterial properties calculation.

In what follows we will amend this limitation of the previous consideration of surface tension contribution to the physical properties of nanomaterials. In general case the surface energy can be written in the form [9]:

$$
E_{\text {surf }}=\int \mu d s
$$

where $\mu$ is the surface tension coefficient and integration is performed over the surface. The small change of the surface area $d s$ can be rewritten by the following way

$$
d s=U_{x x} U_{y y} d x d y
$$

Here $U_{i i}(i=x, y)$ is a component of strain tensor, the normal to the surface is oriented along $z$ axis. Keeping in mind that there is no inversion center near the material surface, the strain tensor component can be related to surface polarization $P_{s z}$ via piezoelectric coefficients $d_{i i}$, namely $U_{i i}=d_{i i z} P_{z}$. Substitution of this relation into Eqs. (1), (2) yields:

$$
E_{\text {surf }}=\int \mu d_{x x z} d_{y y z} P_{s z}^{2} d x d y
$$


Note that Eq. (3) can be used directly for ferroelectric thin films also. Contrary to the films in nanomaterials there is another contribution from surface tension related to the uniform mechanical pressure $p=\mu /\left(R_{1}+R_{2}\right)$, where $R_{1}$ and $R_{2}$ are the main curvature radiuses of the particle surface $\left(R_{1,2} \rightarrow \infty\right.$ for the films). For ferroelectrics without inversion center this pressure induces polarization via piezoelectric coupling. For ferroelectrics with inversion center (e.g. ferroelectrics with perofskite structure) the contribution of the pressure can be expressed via electrostriction and included into free energy on the base of the procedure proposed in [11]. Therefore free energy (with determined stress $\sigma_{i k}=-\delta_{i k} p$, expressed in terms of uniform pressure $p=\mu /\left(R_{1}+R_{2}\right)$, polarization $P_{z}$ and strain $U_{i k}=\delta_{i k} U$ as independent variables) acquires the form:

$$
\begin{aligned}
F= & \int d^{3} r\left[\frac{\alpha\left(T-T_{c}\right)}{2} P_{z}^{2}+\frac{\beta}{4} P_{z}^{4}+\frac{\gamma}{6} P_{z}^{6}+\frac{\delta}{2}\left(\nabla P_{z}\right)^{2}-\frac{3}{2} C_{11} U_{h}^{2}-\right. \\
& \left.-3 C_{12} U_{h}^{2}-Q_{11} U_{h} P_{z}^{2}-2 Q_{12} U_{h} P_{z}^{2}-E_{z} P_{z}-\mu\left(\frac{1}{R_{1}}+\frac{1}{R_{2}}\right) U_{h}\right]+E_{\text {surf }}
\end{aligned}
$$

where $T_{c}$ is ferroelectric transition temperature in a bulk material, $Q_{i j}$ and $C_{i j}$ are respectively electrostriction and elastic coefficients in Voigt presentation, $E_{z}$ is the electric field. For spherical particles $R_{1}=R_{2}=R$. When taking into account the particle spherical symmetry, we assume that the magnitude of oriented along $z$ axis polarization $P(r) \equiv P$ depends only upon the radial position $r$. Analogically to [11 and taking into account the depolarization field energy, one can obtain the renormalized free energy in the form

$$
\begin{aligned}
& F=4 \pi \int r^{2} d r\left[\frac{\widetilde{a}}{2} P^{2}+\frac{b}{4} P^{4}+\frac{c}{6} P^{6}+\frac{\delta}{2}\left(\frac{d P}{d r}\right)^{2}-\left(\frac{1}{2} E_{d}+E_{0}\right) P\right]+8 \pi \int \mu d^{2} P^{2} r d r \\
& \widetilde{a}=\alpha\left(T-T_{c}\right)+\frac{4 \mu}{R} \frac{Q_{11}+2 Q_{12}}{C_{11}+2 C_{12}}, \quad b=\beta+\frac{Q_{11}+2 Q_{12}}{6\left(C_{11}+2 C_{12}\right)}, \quad c=\gamma, \quad
\end{aligned}
$$

Here $E_{0}$ is external electric field, $E_{d}$ is depolarization field $d_{x x z}=d_{y y z} \equiv d$ is inverse piezoelectric effect constant. Hereinafter we will consider ferroelectrics with perofskite structure and nanoparticles of spherical form so we will use the Eq. (4b). 


\section{B. Surface tension}

Let us proceed to the consideration of surface tension coefficient temperature dependence related to phase transition from paraelectric to ferroelectric phase. Critical temperature dependence of surface tension coefficient when approaching the transition from solid state to liquid state is well known [9]. To the best of our knowledge nobody considered influence of structural phase transition like paraelectric-ferroelectric on surface tension. We carried out the calculations in the following model.

In the solids the appearance of polarization manifests itself via the charges on the surface. In nanomaterials and in the films the charges can appear in some region near the surface where polarization is essentially inhomogeneous. It is known that these charges cause the depolarization field in ferroelectrics. In the considered case of single domain nanoparticles the only possibility of depolarization field compensation is the space-charge layer on the surface that has to include the charges of opposite sign to those related to polarization. The possible sources of space-charge layer including free carriers (if any) localized near the surface one can find in ref. [7]. The influence of the flat surface on the aforementioned charges $q$ can be considered by means of the interaction with image charges $q^{*}=q\left(\varepsilon_{i}-\varepsilon_{e}\right) /\left(\varepsilon_{i}+\varepsilon_{e}\right)$ (see, e.g. ref. [12]), where $\varepsilon_{i}$ and $\varepsilon_{e}$ are respectively the dielectric permittivity of the material of particle and surrounding media. All these charges cause the additional energy $w_{s}$ related to the surface [9], so that

$$
\Delta \mu_{s}=n \int_{V} w_{s}(x, y, z) d^{3} r
$$

Here $n$ is the concentration of the charges related to inhomogeneous spontaneous polarization (i.e. it is non-zero only in ferroelectric phase), the integration is performed over the volume of particle. Taking into account Coulomb interaction between the dipole with moment $r_{d} q$ and its image, one obtains the following expression

$$
\Delta \mu_{s}=\frac{n q q^{*}}{\varepsilon_{i}} \int_{a}^{l \gg a}\left(\frac{1}{2 z}+\frac{1}{2 z+2 r_{d}}-\frac{2}{2 z+r_{d}}\right) d z
$$


Here $a, r_{d}$ and $l$ are respectively a lattice constant, minimal distance between positive and negative charges and the size of the region of inhomogeneous polarization. Integration in Eq. (5) yields

$$
\Delta \mu_{s}=\frac{n q^{2}}{2} \frac{\varepsilon_{i}-\varepsilon_{e}}{\varepsilon_{i}\left(\varepsilon_{i}+\varepsilon_{e}\right)} \ln \left(\frac{4 a\left(a+r_{d}\right)}{\left(2 a+r_{d}\right)^{2}}\right)
$$

This relation can be simplified in supposition $r_{d} / a<<1$, so that

$$
\Delta \mu_{s} \approx \frac{n q^{2}}{8} \frac{\varepsilon_{i}-\varepsilon_{e}}{\varepsilon_{i}\left(\varepsilon_{i}+\varepsilon_{e}\right)}\left(\frac{r_{d}}{a}\right)^{2}
$$

It should be noted that the consideration we just have carried out can be applied both for flat surface and for the surfaces with the principal radii of curvature much larger than $r_{d}$ value. This statement follows from the derivation of $\Delta \mu_{s}$ for the case if spherical particle performed in Appendix1.

It should be underlined that since for spherical particles $\Delta \mu_{s}(T) \sim\left(\varepsilon_{i}-\varepsilon_{e}\right) /\left(\varepsilon_{i}\left(\varepsilon_{i}+\varepsilon_{e}\right)\right)$ the temperature dependence (see below) is the same for all the considered forms. Moreover since approximately $r_{d} / a \leq 1$, it is possible to say that the expressions (6a) and (A1-6) coincide with each other. More generally it would be better to rewrite $\Delta \mu_{s}$ in the following form:

$$
\Delta \mu_{s}=\varkappa n q^{2} \frac{\varepsilon_{i}-\varepsilon_{e}}{\varepsilon_{i}\left(\varepsilon_{i}+\varepsilon_{e}\right)}
$$

where the value of the coefficient $\varkappa$ depends on particle shape, its value being $\varkappa \leq 1$.

The surface tension coefficient in ferroelectric phase can represented as the sum of $\Delta \mu_{s}$ and that of paraelectric phase $\mu_{0}$, i.e.

$$
\mu=\mu_{0}+\Delta \mu_{s}
$$

One can see, that while $\mu_{0}$ is temperature independent, $\Delta \mu_{s}$ depends on temperature mainly via $\varepsilon_{i}(T)$. Really in the considered cases of nanomaterials in the form of nanopowders or nanocrystalline ceramics the media surrounding nanoparticles may be considered as nonferroelectric one. In such a case at phase transition temperature $\varepsilon_{i}\left(T_{c r}\right) \rightarrow \infty$ and so 
$\Delta \mu_{s} \rightarrow 0, \mu \rightarrow \mu_{0}$ (see (6), (7)). Note, that in the case of two ferroelectric media (e.g. $\mathrm{BaTiO}_{3}$ particles dispersed in $\mathrm{SrTiO}_{3}$ or $\mathrm{PbZr}_{1-x} \mathrm{Ti}_{x} \mathrm{O}_{3}$ media) both $\varepsilon_{i}$ and $\varepsilon_{e}$ depend on $T$. If the transition temperature in surrounding media $T=T_{e c}$, in these composite materials $\Delta \mu_{s} \rightarrow-n q^{2} / \varepsilon_{i}\left(r_{d} / a\right)^{2}$ at $T \rightarrow T_{e c}$. Note, that the negative sign of $\Delta \mu_{s}$ can be valid in some temperature region where $\varepsilon_{e}>\varepsilon_{i}$ in both aforementioned cases. Keeping in mind that surface tension must be positive, it is necessary to add the inequality $\left|\Delta \mu_{s}\right| / \mu_{0}<1$ to Eq. (7).

Hereinafter we consider only the case of nonferroelectric surrounding media.

\section{DEPOLARIZATION FIELD EFFECT}

We will consider this effect in the model described in previous section. Namely the charges localized in the thin surface layer, where polarization is the most inhomogeneous, create depolarization field inside the particle. This field can be compensated by space-charge layer on the surface (see Section 2).

The magnitude of depolarization field was calculated on the base of equation written in ref. [12] for homogeneously polarized dielectric submerged in a medium with dielectric susceptibility $\varepsilon_{e}$. To take into account additional charges related to inhomogeneous polarization and those from space-charge layer we added the term $-4 \pi n_{\alpha} P_{V \alpha}$. In the case of external field absence this yields

$$
\left[\left(1-n_{\alpha}\right) \varepsilon_{e}+n_{\alpha}\right] E_{d \alpha}+4 \pi n_{\alpha}\left(P_{\alpha}-P_{V \alpha}\right)=0, \alpha=x, y, z
$$

where $E_{d}$ is depolarization field, $n_{\alpha}$ is depolarization factor, that depends on the sample form and for spherical form $n_{\alpha}=1 / 3$ (see e.g. ref. [12]), all the quantities $E_{d \alpha}, P_{\alpha}, P_{V \alpha}$ being dependent on the coordinates of considered point $\vec{r}$. The $P_{V \alpha}$ will be considered as variational parameter to minimize the energy related to depolarization field.

With respect to Eq. (8) this energy can be written as

$$
G=\frac{1}{V} \frac{1}{2} \sum_{\alpha} \int E_{d \alpha}^{2} d V=\frac{8 \pi^{2}}{V} \sum_{\alpha}\left(\frac{n_{\alpha}}{\left(1-n_{\alpha}\right) \varepsilon_{e}+n_{\alpha}}\right)^{2} \int\left(P_{\alpha}-P_{V \alpha}\right)^{2} d V
$$


Equilibrium value of $P_{V \alpha 0}$ can be obtained from the condition $\frac{\delta G}{\delta P_{V \alpha}}=0$, that yields

$$
P_{V \alpha 0}=\frac{1}{V} \int d V P_{\alpha}(\vec{r}) \equiv \bar{P}_{\alpha}
$$

where $\bar{P}$ is average polarization.

The Eq. (8) with respect to Eq. (10) gives the following equation for depolarization field:

$$
E_{d \alpha}=-\frac{4 \pi n_{\alpha}\left(P_{\alpha}-\bar{P}_{\alpha}\right)}{\left(1-n_{\alpha}\right) \varepsilon_{e}+n_{\alpha}}
$$

For single domain spherical nanoparticle with polarization along $z$ axis (i.e. $\alpha=z$, $\left.n_{z}=1 / 3\right) \mathrm{Eq} .(11)$ gives

$$
E_{d z}=-a_{0}\left(P_{z}-\bar{P}_{z}\right), a_{0} \equiv \frac{4 \pi}{1+2 \varepsilon_{e}}
$$

Note, that for the film where $z$ coincides with normal to the film surface $n_{z}=1$ [12], so that Eq. (11) leads to the expression obtained in ref. [6] for short circuited film: $E_{d z}=$ $-4 \pi\left(P_{z}-\bar{P}_{z}\right)$. The later is related to the fact that both the short circuit condition and the

charges on the surface produce similar potential distribution which is able to compensate the depolarization field completely (when polarization is homogeneous, i.e. $P_{z}=\bar{P}$ ) or partly (when polarization is inhomogeneous). Note, that Eq. (11) makes it possible to calculate the depolarization field for nanoparticles of different form for which depolarization factors $n_{\alpha}$ are known. In particular for the particle of cylindrical form with $z$ along the axis of rotation $n_{z}=0$, i.e. $E_{d z}=0$.

\section{FREE ENERGY FUNCTIONAL AND EULER-LAGRANGE EQUATION FOR POLARIZATION}

\section{A. Free energy}

Allowing for the results of previous sections for depolarization field contribution (see Eq. (12)), we can write the free energy (4b) in the following form: 


$$
\begin{gathered}
F=\frac{1}{V}\left(4 \pi \int_{0}^{R} r^{2} d r\left[\frac{a}{2} P^{2}+\frac{b}{4} P^{4}+\frac{c}{6} P^{6}+\frac{\delta}{2}\left(\frac{d P}{d r}\right)^{2}-\left(\frac{a_{0}}{2} \bar{P}+E_{0}\right) P\right]+8 \pi \int_{0}^{R} \mu d^{2} P^{2} r d r\right) \\
a=\alpha\left(T-T_{c}\right)+\frac{4 \mu}{R} \frac{Q_{11}+2 Q_{12}}{C_{11}+2 C_{12}}+a_{0}
\end{gathered}
$$

Having written Eq. (13) we take into account spherical symmetry and that is why the magnitude of oriented along $z$ axis polarization $P(r) \equiv P$ depends only upon the radial position $r$. By virtue of surface tension temperature dependence (see Eqs. (6), (7)) additional terms with temperature dependence appear in surface energy (see last term in Eq. (13)) and in the coefficient before $P^{2}$ (see Eq. (14)). Since $\mu>0$ and $Q_{11}+2 Q_{12}>0$ in perofskites both the second term in $a$ (related to surface tension) and the third one (related to depolarization field) tends to decrease $T_{c}$ value, i.e. to destroy ferroelectric phase transition.

\section{B. Euler-Lagrange equation}

Variation of free energy functional (13) yields the following Euler-Lagrange equation for static polarization distribution and the boundary condition:

$$
\begin{aligned}
a P+b P^{3}+c P^{5}-\delta\left(\frac{d^{2} P}{d r^{2}}+\frac{2}{r} \frac{d P}{d r}\right) & =E_{0}+a_{0} \bar{P} \\
\left(\lambda \frac{d P}{d r}+P\right)_{r=R} & =0 \\
\lambda & =\frac{\delta}{\mu d^{2}}
\end{aligned}
$$

It follows from Eq. (15c), that extrapolation length $\lambda$ depends on surface tension coefficient $\mu(T)$ given by Eqs. (6), (7), so that $\lambda$ has to be temperature dependent quantity. On the other hand $\mu>0$ in all the materials [9], $d^{2}>0$ so the sign of $\lambda$ defines by that of $\delta$. In what follows we will consider the case $\delta>0$ that leads to positive extrapolation length $\lambda>0$. Note that in the case $\delta<0$ one has to add the fourth power of gradient into free energy expansion to conserve the system stability. It is seen that it is possible to control 
extrapolation length magnitude (i.e. boundary condition) by the choice of the materials with the smaller or larger piezoelectric constant and surface tension.

\section{SIZE DRIVEN FERROELECTRIC PHASE TRANSITION}

Let us begin with the solution of Eq. (15a) with boundary condition (15b) in paraelectric

phase where the polarization induced by external electric field can be small enough, so that the nonlinear terms in Eq. (15a) can be neglected. In such a case the substitution of $P=\widetilde{P} / r$ transforms Eqs. (15a), (15b) to the following forms:

$$
\begin{array}{r}
a \frac{\widetilde{P}}{r}-\delta \frac{1}{r} \frac{d^{2} \widetilde{P}}{d r^{2}}=E_{0}+a_{0} \bar{P} \\
\left.\lambda \frac{d \widetilde{P}}{d r}\right|_{r=R}+\left.\left(1-\frac{\lambda}{R}\right) \widetilde{P}\right|_{r=R}=0
\end{array}
$$

Keeping in mind, that in the particle center polarization $P(0)$ is finite, one can easily obtain the solution of Eq. (16a) and write $P(r)$ as

$$
\begin{aligned}
P(r) & =\frac{1}{a}\left(E_{0}+a_{0} \bar{P}\right)\left[1-\frac{R}{r} \frac{\operatorname{sh}(r \sqrt{a / \delta})}{M(R)}\right] \\
M(R) & =\lambda \sqrt{\frac{a}{\delta}} \operatorname{ch}\left(R \sqrt{\frac{a}{\delta}}\right)+\left(1-\frac{\lambda}{R}\right) \operatorname{sh}\left(R \sqrt{\frac{a}{\delta}}\right)
\end{aligned}
$$

Averaged polarization can be obtained by the integration of (17a) over particle volume, i.e. $\bar{P}=3 / R^{3} \int_{0}^{R} P(r) r^{2} d r$, that yields

$$
\begin{aligned}
\bar{P}(R) & =\frac{E_{0} W(R)}{a-a_{0} W(R)} \\
W(R) & =1-\frac{3}{R^{2}} \frac{\delta}{a} \frac{R \sqrt{a / \delta} \operatorname{ch}(R \sqrt{a / \delta})-\operatorname{sh}(R \sqrt{a / \delta})}{M(R)}
\end{aligned}
$$

Substituting Eq. (18a) into (17a), one obtains

$$
P(r)=\frac{E_{0}}{a-a_{0} W(R)}\left[1-\frac{R}{r} \frac{\operatorname{sh}(r \sqrt{a / \delta})}{M(R)}\right]
$$

It is clearly seen that polarization in paraelectric phase is proportional to external field, the coefficient of proportionality being the inhomogeneous dielectric susceptibility 


$$
\chi_{P E}(r)=\frac{1}{a-a_{0} W(R)}\left[1-\frac{R}{r} \frac{\operatorname{sh}(r \sqrt{a / \delta})}{M(R)}\right]
$$

It is follows from Eqs. (18a,b) and (14) that for bulk material $(R \rightarrow \infty) W(R) \rightarrow 1$ and $\bar{P}(R) \rightarrow E_{0} /\left(\alpha\left(T-T_{c}\right)\right)$ as it has to be.

The average value of susceptibility can be extracted from Eq. (18a) as the coefficient before $E_{0}$. It follows from Eq. (18a) that the susceptibility becomes infinitely large at the condition:

$$
a-a_{0} W(R)=0
$$

Allowing for that both $a$ and $W(R)$ depend on particle radius $R$ and temperature $T$, the condition $(19 \mathrm{c})$ can be satisfied at some critical radius $R_{c r}$ (temperature is fixed) or at some critical temperature $T_{c r}$ (radius is fixed). These critical values correspond to size driven ferroelectric phase transition in nanomaterials. Its consideration without contribution of depolarization field and surface tension was carried out earlier in e.g. ref. [4], [5], [13], [14. Comparative analysis of the results with and without these factors contribution will be performed later.

Because of complex enough form of $W(R)$, that depends also on $T$ via temperature dependence of parameter $a$ (see Eq. (14)) it appeared cumbersome to calculate $T_{c r}$ and $R_{c r}$ analytically in general case. Because of this we performed the calculation numerically introducing the following dimensionless parameters and functions:

$$
\begin{aligned}
& R_{0}=\sqrt{\frac{\delta}{4 \pi}}, \quad \theta_{0}=\frac{\alpha T_{c}}{4 \pi}, \quad \rho_{0}=\frac{\mu_{0}}{\pi R_{0}}\left(\frac{Q_{11}+2 Q_{12}}{C_{11}+2 C_{12}}\right), \\
& \lambda_{0}=\frac{\sqrt{4 \pi \delta}}{\mu_{0} d^{2}}, \quad \eta=\frac{\varkappa n q^{2}}{\mu_{0}}, \quad \Delta \mu_{F E}(T, R)=\frac{\varepsilon_{i}-\varepsilon_{e}}{\varepsilon_{i}\left(\varepsilon_{i}+\varepsilon_{e}\right)} .
\end{aligned}
$$

The results of $T_{c r}$ calculations is represented in Fig. 1.

The increase of $\mu_{0}$ value at other fixed parameters leads to the decrease $\lambda_{0}$, that results in critical temperature decrease and in critical radius increase (compare the curves 1 and 2 and their crossing points with abscissa axis). Also one can see, that the decrease of surrounding medium dielectric permittivity $\varepsilon_{e}$ leads to $R_{c r}$ decrease and $T_{c r}$ increase, these 
effects being larger for smaller $\lambda_{0}$ value. Dashed-dotted curves correspond to approximate analytical formulas obtained by the following way.

Estimations have shown, that for $\varepsilon_{e} \leq 100$ and $R \geq 10 \mathrm{~nm}$ the contribution of $a_{0}$ into $a$ value is the largest one, i.e. further we can put $a \approx a_{0}$. Keeping in mind that $\sqrt{\delta / a}$ can be estimated as correlation radius value, that has to decrease due to depolarization field contribution, it is reasonable to suppose that $\sqrt{\delta / a_{0}}$ value is about several lattice constants. Therefore for $R \sqrt{a_{0} / \delta}>3 \div 5$ one can derive from (19c), allowing for that at transition point $\mu \rightarrow \mu_{0}$, the following approximate analytical expression for the critical temperature of size driven phase transition:

$$
T_{c r}(R) \approx T_{c}\left(1-\frac{R_{L}}{R}-\frac{R_{\lambda}}{R-R_{c}}\right)
$$

Here we introduce the parameters

$$
R_{c}=\frac{R_{0} \lambda_{0} \sqrt{4 \pi}}{\lambda_{0} \sqrt{a_{0}}+\sqrt{4 \pi}}, \quad R_{L}=\rho_{0} \frac{R_{0}}{\theta_{0}}, \quad R_{\lambda}=\frac{R_{0}}{\theta_{0}}\left(\frac{3 \sqrt{a_{0}}}{\lambda_{0} \sqrt{a_{0}}+\sqrt{4 \pi}}\right) .
$$

$R_{c}$ can be considered as correlation radius renormalized by contribution of depolarization field and surface tension. $R_{L}$ is correlation radius renormalized only by surface tension.

For arbitrary $T<T_{c}$ approximate formula for critical radius $R_{c r}(T)$ can be obtained from Eq. (21a), as the solution of the quadratic equation with respect to $T_{c r}=T,{ }_{-} R=R_{c r}$. The analytical expression can be obtained for the some cases.

In particular, for the large enough particles when $R \sqrt{a_{0} / \delta}>>1$ and $R / R_{c}>>1$, one can neglect $R_{c}$ in the denominator of the third term of Eq. $(21 \mathrm{a})$ and formula for $T_{c r}(R),{ }_{-} R_{c r}(T)$ dependences can be written as

$$
\begin{aligned}
& T_{c r}(R) \approx T_{c}\left(1-\frac{R_{L}+R_{\lambda}}{R}\right)=T_{c}\left(1-\frac{R_{c r}(T)}{R}\left(1-T / T_{c}\right)\right), \\
& R_{c r}(T) \approx \frac{R_{0}}{\theta_{0}\left(1-T / T_{c}\right)}\left(\rho_{0}+\frac{3 \sqrt{a_{0}}}{\lambda_{0} \sqrt{a_{0}}+\sqrt{4 \pi}}\right) .
\end{aligned}
$$

If $R_{L}<<R_{\lambda}$ and $R_{\lambda} /\left(1-T / T_{c}\right)<<R_{c}$ approximate formula for $T_{c r}(R), R_{c r}(T)$ dependences can be written as:

$$
T_{c r}(R) \approx T_{c}\left(1-\frac{R_{\lambda}}{R-R_{c r}}\right), \quad R_{c r} \approx R_{c}
$$


Thus, in this particular temperature range critical radius does not depend on temperature. It is seen from $(21 \mathrm{~b})$ that $\left(T_{c r}-T_{c}\right)$ is inversely proportional to the particle size and e.g. for ferroelectrics with perofskite structure $T_{c r}<T_{c}$ because $Q_{11}+2 Q_{12}>0$ (although $\left.Q_{12}<0\right)$. It should be noted that the value of $\left(T_{c r}-T_{c}\right)$ is proportional to the coefficient of surface tension, the first and the second term in the brackets being related to surface tension and surface energy respectively. One can see, that Eq. (21b) describes the main features of $T_{c r}$ behavior: $T_{c r}=T_{c}$ at $\mu_{0}=0, T_{c r}$ decreases with $\mu_{0}$ increases and $T_{c r}$ value increases with the particle size increases $\left(T_{c r} \rightarrow T_{c}\right.$ at $\left.R \rightarrow \infty\right)$. The results of more detailed calculation of $T_{c r}(R)$ dependence given by Eq. (21b) is shown in Fig.1 by dashed-dotted line. One can see that the coincidence between calculations on the base of Eq. (19c) and on the base of approximate formula (21b) is very good for $\lambda_{0}=250$, for $\lambda_{0}=25$ the $T_{c r}(R)$ dependences given by curve 1 and dash-dotted line are similar, however quantitative values of $T_{c r}$ for both curves are different.

The equation (21b) at $T=0$ does correspond to the crossing points of the curves in Fig. 1 with abscissa axis. But it is possible to obtain $R_{c r}$ at any fixed arbitrary temperature with the help of Eq. (21b).

It follows from Eqs. (21) that critical parameters of size driven ferroelectric phase transition in nanomaterials are defined completely by physical characteristics of the material such as Curie-Weiss constant $C_{W}=4 \pi / \alpha$, electrostriction constants $Q_{i j}$, elastic modulus $C_{i j}$, piezoelectric constant $d_{1 z} \equiv d$ and surface tension coefficient $\mu_{0}$. This appeared possible due to the fact that extrapolation length that usually is a phenomenological parameter was expressed via $\mu_{0}$ and $d$ coefficients and surface tension was related to polarization via electrostriction effect (see section 2). 


\section{PROPERTIES IN FERROELECTRIC PHASE AND FREE ENERGY WITH RENORMALIZED COEFFICIENTS}

In ferroelectric phase $R>R_{c r}, T<T_{c r}$ the nonlinear term in Eqs. (13), (15) can not be neglected. The simplest way to take it into account is to look for polarization on the base of direct variational method. We choose solution (19a) as a trial function and amplitude factor will be treated as variational parameter, therefore

$$
P^{F E}=P_{V}\left(1-\frac{R}{r} \frac{\operatorname{sh}(r \sqrt{a / \delta})}{M(R)}\right)
$$

Here $P_{V}$ is variational parameter that represents the amplitude of polarization space distribution.

After substitution of the trial function (22) into integral (13) one can obtain the free energy in the following form:

$$
F=\frac{A_{R}}{2} P_{V}^{2}+\frac{B_{R}}{4} P_{V}^{4}+\frac{C_{R}}{6} P_{V}^{6}-E_{R} P_{V}
$$

Here

$$
\begin{gathered}
A_{R}=\frac{3}{R^{3}} \int_{0}^{R} r^{2} d r\left[a\left(1-\frac{R}{r} \frac{s h(r \sqrt{a / \delta})}{M(R)}\right)^{2}+\delta\left(\frac{d}{d r}\left(\frac{R}{r} \frac{s h(r \sqrt{a / \delta})}{M(R)}\right)\right)^{2}\right]- \\
\quad-a_{0}(W(R))^{2}+\frac{\delta}{\lambda} \frac{3}{R}\left(1-\frac{s h(R \sqrt{a / \delta})}{M(R)}\right)^{2} \\
B_{R}=b \frac{3}{R^{3}} \int_{0}^{R} r^{2} d r\left(1-\frac{R}{r} \frac{\operatorname{sh}(r \sqrt{a / \delta})}{M(R)}\right)^{4}, \quad C_{R}=c \frac{3}{R^{3}} \int_{0}^{R} r^{2} d r\left(1-\frac{R}{r} \frac{\operatorname{sh}(r \sqrt{a / \delta})}{M(R)}\right)^{6} \\
E_{R}=E_{0} W(R),
\end{gathered}
$$

where parameters $a, b$ and $c$ are given by Eq. (14).

Keeping in mind, that average polarization is $\bar{P}=P_{V} W(R)$, we can rewrite Eq. (23a) as follows

$$
F=\frac{A_{R}}{W^{2}(R)} \frac{\bar{P}^{2}}{2}+\frac{B_{R}}{W^{4}(R)} \frac{\bar{P}^{4}}{4}+\frac{C_{R}}{W^{6}(R)} \frac{\bar{P}^{6}}{6}-\bar{P} E_{0}
$$


It is worth to underline that the terms in Eq. (13) which correspond to polarization gradient, depolarization field and surface energy contribute to $A_{R}$ (see respectively the second, the third and the forth terms in $A_{R}$ ). Therefore the main peculiarities of nanomaterials properties is expected to be related to the first term in Eqs. (23a), (23c).

It is obvious that Eqs. (23a) and (23c) have the from of power series of the amplitude of the polarization space distribution and average polarization respectively. In general case all the coefficients (23b) depend on a particle size and temperature.

The main advantage of the Eqs. (23a,c), that they make it possible to obtain $P_{V}$ and $\bar{P}$ by conventional minimization procedure. In particular for the phase transitions of the second order

$$
P_{V}=\sqrt{-\frac{A_{R}}{B_{R}}}, \quad \bar{P}=W(R) \sqrt{-\frac{A_{R}}{B_{R}}}
$$

and the polarization profile can be easily obtained with the help of Eq. (22).

The physical properties e.g. dielectric susceptibility $\chi(r), \bar{\chi}$ and pyrocoefficient $\Pi(r), \bar{\Pi}$ can be calculated as the derivative of polarization over $E_{0}$ and $T$ respectively.

The results of calculations of average dielectric susceptibility dependence on the particle size and temperature are depicted in Figs. 2 and 3 respectively both for paraelectric $(R<$ $\left.R_{c r}, T>T_{c r}\right)$ and ferroelectric $\left(R>R_{c r}, T<T_{c r}\right)$ phases for different values of dimensionless extrapolation length $\lambda_{0}$. It is seen that inverse susceptibility linearly depends on $T$ like that in Curie-Weiss (C-W) law (see Fig. 3), although in ferroelectric phase there is small deviation from linearity for small extrapolation length (see curves 1). In paraelectric phase $1 / \bar{\chi} \sim\left(R_{c r} / R-1\right)$ while in ferroelectric phase the dependence of $\bar{\chi}$ on $R^{-1}$ declines from linearity more strongly, especially for $\mu(T) / \mu_{0}-1=\eta \Delta \mu_{F E}(T) \neq 0$ (see solid curve 2), although general view resembles that of $\mathrm{C}-\mathrm{W}$ law (see inset to Fig. 2) with maximum at $R=R_{c r}$. It follows from Figs. 2,3 that at $\mu_{0}=0 \bar{\chi}=\chi_{b}$. (because at $\mu_{0} \rightarrow 0 \lambda_{0} \rightarrow \infty$, that corresponds to the bulk) and the decrease of $\eta$ value makes the dependences linear, i.e. $1 / \bar{\chi} \sim$ $\left(R_{c r} / R-1\right)$ and $1 / \bar{\chi} \sim\left(T-T_{c}\right)$ (see dashed curves in the Figs.). Thus all deviation from linearity in ferroelectric phase is related to $\Delta \mu_{F E}(T)$ contribution. The influence of dielectric 
permittivity $\varepsilon_{e}$ of the surrounding medium appeared to be strong enough (compare solid and dotted curves in Figs. 2,3). This effect is related to the contribution of depolarization field and temperature dependent part of surface tension.

Practically the same type of influence of $\mu_{0}, \Delta \mu$ and $\varepsilon_{e}$ on the averaged spontaneous polarization one can see from Figs. 4 and 5, where the temperature and size dependence of averaged polarization are depicted, the crossing points of the curves with abscissa axis in Figs. 4 and 5 being respectively $R_{0} / R_{c r}$ and $T_{c r} / T_{c}$ values. One can see, that the dependence of this quantities on extrapolation length is a good agreement with approximate formulas $(21 \mathrm{~b}, \mathrm{c})$, where the second term in the brackets reflects the influence of extrapolation length i.e. the larger $\lambda_{0}$ the larger $T_{c r}$ and the smaller $R_{c r}$ value has to be. This behavior is related to the fact, that larger extrapolation length corresponds to more homogeneous polarization like that in bulk material, so that the closeness of $T_{c r}$ to $T_{c}$ has to be expected. It is evident, that the destruction of such "strong" polarization can be achieved for small enough particles, i.e. for small $R_{c r}$ value. It is worth to underline, that the calculation of $T_{c r}$ and $R_{c r}$ with the help of Eqs. (21b,c) leads to the following values: $T_{c r}=0.44 T_{c}, R_{c r}=0.036 R_{0}$ (for $\left.\lambda_{0}=25\right), T_{c r}=0.755 T_{c}, R_{c r}=0.0785 R_{0}($ for $\lambda=250)$, which are cloth to those obtained from crossing points in Figs. 4, 5. This speaks in favor of statement that the accuracy of formulas $(21 \mathrm{~b}, \mathrm{c})$ is not bad at all.

The calculations of space distribution of polarization (see Eqs. (22), (24)) had shown, that the contribution of temperature dependent part of surface tension can be large enough (compare solid and dashed lines in Fig. 6). It is seen, that $\eta \Delta \mu_{F E}(T) \neq 0$ flattens the curves and decreases polarization, these two effects can be related to increase of extrapolation length and surface tension respectively with $\mu(T) / \mu_{0}=1+\eta \Delta \mu_{F E}$ increase. Therefore the influence of surface tension and its change under externally controllable parameters (e.g. temperature) can manifest itself mainly in space distribution of physical quantities, the smaller the particle or extrapolation length the larger the effect can be (see Fig. 6).

The considered temperature and size dependences of properties were calculated numerically because of the complex form of the coefficients (23b). These dependences can be 
more clearly seen after analytical calculation of integrals (23b). In particular for $A_{R}$ the integration yields :

$$
A_{R}=\left[a-a_{0} W(R)\right] W(R) .
$$

It is seen that expression in the brackets completely coincides with left hand side of Eq. (19c), so that at $T=T_{c r}$ or $R=R_{c r} A_{R}=0, P_{V}=0, \bar{P}=0$ as it has to be.

The expressions for $B_{R}$ and $C_{R}$ appeared to be very complex (see Appendix 2). But all the coefficients can be simplified allowing for the estimations made in previous section for $T_{c r}$ and $R_{c r}$ approximate calculations. Since the accuracy of these formulas was shown to be good, it is worth to write the approximate formulas for the coefficients $A_{R}, B_{R}$ and then for the physical properties. Allowing for the approximate expression for $W(R)$ at $R \sqrt{a_{0} / \delta}>3 \div 5$ one can rewrite $A_{R}$ approximately in the following form:

$$
A_{R} \approx \alpha\left(T-T_{c}\right)+\frac{\mu}{R}\left[4 \frac{Q_{11}+2 Q_{12}}{C_{11}+2 C_{12}}+3 \frac{d^{2} \sqrt{\delta a_{0}}}{d^{2} \mu_{0}+\sqrt{\delta a_{0}}}\right]
$$

Substitution of Eqs. (21b) for $T_{c r}$ or $R_{c r}$ into Eq. (26a) yields

$$
\begin{aligned}
& A_{R}=\alpha\left(T-T_{c r}+\frac{\Delta \mu_{s}}{\mu_{0}}\left(T_{c}-T_{c r}\right)\right), \\
& A_{R}=\alpha\left(T-T_{c}\right)\left(1-\frac{\mu_{0}+\Delta \mu_{s}}{\mu_{0}} \frac{R_{c r}}{R}\right) .
\end{aligned}
$$

One can see, that for the case when $\Delta \mu_{s} / \mu_{0} \ll 1$ expression (26b) corresponds to that for $\mathrm{C}-\mathrm{W}$ law, and (26c) contains the simple multiplier $\left(1-R_{c r} / R\right)$. But in general case situation is more complicated. Indeed, because $\Delta \mu_{s}$ depends on the material dielectric permittivity (see Eq. (6a)) it can be expressed via $A_{R}$. In particular it is possible when $\left(\varepsilon_{i}-\varepsilon_{e}\right) /\left(\varepsilon_{i}\left(\varepsilon_{i}+\varepsilon_{e}\right)\right) \approx 1 / \varepsilon_{i}$, that is proportional to $A_{R}$. In such a case (26b) and (26c) can be represented as

$$
\begin{gathered}
A_{R}=\alpha\left(T-T_{c r}\right) A_{R 1} \\
A_{R}=\alpha\left(T-T_{c}\right)\left(1-\frac{R_{c r}}{R}\right) A_{R 2}
\end{gathered}
$$




$$
A_{R 1}=\frac{1}{1-\alpha \eta\left(T_{c}-T_{c r}\right)}, \quad A_{R 2}=\frac{1}{1+\alpha\left(T-T_{c}\right) \eta R_{c r} / R}
$$

Allowing for that e.g. for $\mathrm{BaTiO}_{3} \eta \approx 10^{2}$ and $\alpha \sim 10^{-5}$ one can see, that $A_{R 1} \approx 1$ and $A_{R 2} \approx 1$ although the accuracy of this estimation for $A_{R 1}$ is better than for $A_{R 2}$. In what follows we will use Eqs. (27a), (27b) with $A_{R 1}=A_{R 2}=1$.

The formulas (27a) and (27b) can be applied respectively to study the temperature dependence for some fixed radius of the particles and to study the size dependence at some arbitrary fixed temperature.

Keeping in mind, that in adopted approximation $a \approx a_{0}$ and so it is independent on temperature, the coefficient $B_{R}$ also independent on $T$ (see Eq. (23b) and Appendix 2), so that the properties temperature dependence defines completely by (27a). Moreover, because $W(R) \approx 1$, so $P_{V} \approx \bar{P}$ (see Eq. (24)) and $E_{R} \approx E_{0}$ (see Eq. (23b)). In such a case the susceptibility has the conventional form

$$
\bar{\chi}(T)=\frac{1}{\alpha\left(T-T_{c r}\right)}, \quad T>T_{c r}
$$

and

$$
\bar{\chi}(T)=\frac{1}{2 \alpha\left(T_{c r}-T\right)}, \quad T<T_{c r}
$$

respectively for paraelectric and ferroelectric phase of size driven transition. The temperature dependences given by Eqs. (27a), (28a), (28b) correspond completely to those in bulk materials but with substitution $T_{c r}$ for $T_{c}$. These dependences are shown in the Fig. 3 by dash-dotted curves. One can see, that the approximate formulas describe the accurate curves good enough, especially curves numbered by 2 , where dash-dotted curve completely coincides with solid curve, although the coincidence is not so good for the curves 1 . Taking into account, that for ferroelectrics $\varepsilon_{i} \approx 4 \pi \chi$, it is easy to obtain after substitution of $T_{c r}\left(R, R_{c r}\right)$ from Eqs._(21b) into Eq..(28), that at fixed temperature $T$ the dependence of nanoparticle dielectric permittivity over particle radius $R$ can be represented as:

$$
\begin{aligned}
& \overline{\varepsilon_{i}}(T, R)=\frac{\varepsilon_{b}}{2\left(1-R_{c r}(T) / R\right)}, \quad R>R_{c r}(T), \\
& \overline{\varepsilon_{i}}(T, R)=\frac{\varepsilon_{b}}{\left(R_{c r}(T) / R-1\right)}, \quad R<R_{c r}(T) .
\end{aligned}
$$


Here $\varepsilon_{b}=C_{W} /\left(T_{c}-T\right)$.

The spontaneous polarization approximate formulas can be obtained from Eq. (24) at $W(R) \approx 1$ and $B_{R} \approx \beta$ (see Appendix 2). This yields for average polarization

$$
\bar{P}=\sqrt{\frac{\alpha}{\beta}\left(T_{c r}-T\right)}
$$

This formula completely coincides with that for bulk material when substituting $T_{c r}$ for

$T_{c}$. The approximate expression (29) describes exact curves $\bar{P}(T)$ not bad at all (compare solid and dash-dotted curves in Fig. 5 which completely coincide for $\lambda_{0}=250$ ). Note, that these curves describe also the temperature dependence of inverse pyrocoefficient $\bar{\Pi}$ because

$$
\bar{\Pi}=\frac{d \bar{P}}{d T}=-\frac{\alpha}{\beta} \frac{1}{\bar{P}}
$$

For the phase transitions of the first order the spontaneous polarization, the dielectric susceptibility and the pyrocoefficient depend also on $C_{R}$ coefficient given by (23b). It was shown (see Appendix 2) that $C_{R} \approx c$, so that all the physical quantities for nanomaterials can be described by the same formulas as for bulk materials, but with the coefficient before $P^{2}$ in the form of (27a) or (27b). The substitution of Eq. (27b) will make it possible to describe approximately size dependence of the properties. The description on the base of the approximate formulas appeared to be good enough especially for the curves 2 (compare solid and dash-dotted curves in Figs. 2, 4).

The space distribution of the properties in nanomaterial can be calculated with the help of Eq. (22). Since in adopted approximation $a \approx a_{0}$ is temperature independent quantity and $\bar{P}=P_{V}$ the properties profiles will be described by the expression in the brackets of Eq. (22), i.e. their form will be the same as that depicted in Fig. 6.

\section{THE DEPOLARIZATION FIELD AND SURFACE TENSION COEFFICIENT SIZE AND TEMPERATURE DEPENDENCIES}

In this section we will consider in details size and temperature effects in depolarization field and surface tension coefficient. 


\section{A. The depolarization field}

It was shown in Sect. 3, that for nanoparticles with spherical form the depolarization field is given by Eq. (12) in the model, when it can be partly compensated by space-charge layer. If dielectric permittivity of surrounding media $\varepsilon_{e}$ independent on $T$, temperature dependence of $E_{d z} \equiv \dot{E}_{d}$ will be related to that of $\left(-P_{z}+\bar{P}_{z}\right) \equiv(-P+\bar{P})$. Allowing for space distribution $P(r)$ (see Eq. (22)) one can calculate $E_{d}(r)$ distribution, which is depicted in the inset to Fig. 6. It is seen that for infinitely large extrapolation length $\left(\lambda_{0} \sim 1 / \mu_{0}, \mu_{0}=0\right)$ that corresponds to practically homogeneous polarization, $E_{d}=0$. For smaller extrapolation length values when polarization is inhomogeneous $E_{d}<0$ inside the particle and transforms into $E_{d}>0$ near the surface. The absolute values of $E_{d}$ decrease with $\lambda_{0}$ increase (compare the curves 1 and 2 in the inset to Fig.6). Therefore depolarization field tends to decrease the polarization inside the particle and to increase it near the surface. Since in the considered case of positive extrapolation length polarization near the surface is smaller than inside the particle, depolarization field tends to flatten the polarization in nanoparticle, i.e. to make it more homogeneous.

\section{B. Surface tension coefficient}

Let us discuss now the size and temperature dependence of surface tension coefficient in ferroelectric nanomaterials. In the adopted model surface tension coefficient is given by Eqs. (7), (6), so that its size and temperature dependences are defined by that of nanoparticle dielectric permittivity $\varepsilon_{i}$ in supposition that permittivity of the surrounding media $\varepsilon_{e}$ is temperature independent quantity. Because of $\varepsilon_{i}$ dependence on the temperature $T$ and particle radius $R$ the quantities $\Delta \mu_{s}$ and $\Delta \mu_{F E}$ from Eqs. (6), (20c) have to depend on these parameters. One can see the dependence $\Delta \mu_{F E}(T)$ in Fig. 7 , and $\Delta \mu_{F E}(R)$ in Fig.8 for the several values of $\varepsilon_{e}$. Negative sign of $\Delta \mu_{F E}$ corresponds to the case $\varepsilon_{i}<\varepsilon_{e}$, that

realizes at $\varepsilon_{e} \geq 300$ both for $\lambda_{0}=25$ and 250 (see inset). The existence of the maximum in 
curve 3 is related to the temperature region where $\varepsilon_{i}$ and $\varepsilon_{e}$ is close to one another. Positive sign of $\Delta \mu_{F E}$ corresponds to the case $\varepsilon_{i}>\varepsilon_{e}$ (see curves 1,2$)$, the curve 1 being very close to the respective curve for $1 / \bar{\chi}$ dependences on temperature (see Fig.3) and on the particle radius (see inset to Fig. 8).

One can see from Figs.7, 8 that $\left|\Delta \mu_{F E}\right| \sim 10^{-3} \div 10^{-2}$, i.e. the relative contribution of $\Delta \mu_{s}$ to the surface tension $\mu$ is equals $\Delta \mu_{s} / \mu_{0}=\eta \Delta \mu_{F E} \sim 10^{-1} \div 1$, for $\eta=100$ that we used when plotting all the Figures 1-6.

Therefore for nanomaterials $\Delta \mu_{s}(T)$ can be both positive and negative quantity, its absolute value $\left|\Delta \mu_{s}(T)\right| \leq \mu_{0}$. This different possibilities depend on the material characteristics (particles size, extrapolation length, transition temperature in bulk) and dielectric constant of the surrounding media.

The temperature dependence of surface tension coefficient results in extrapolation radius temperature dependence because $\lambda \sim 1 / \mu$ (see Eq. (15c)). Since in paraelectric phase $\mu=\mu_{0}$ is temperature independent quantity, we depicted $\lambda / \lambda_{P E}=1 /\left(1+\Delta \mu_{s} / \mu_{0}\right)$ in Fig. 9 as the function of temperature. Note, that $\lambda_{P E}=R_{0} \lambda_{0}$. One can see, that in paraelectric phase $\left(T \geq T_{c r}\right) \lambda / \lambda_{P E}=1$, while in ferroelectric phase $\left(T<T_{c r}\right)$ its behavior depends strongly

on $\varepsilon_{e}$ value as it has been already discussed above, allowing for $\Delta \mu_{s} / \mu_{0}=\eta \Delta \mu_{F E}$. The same type behavior was obtained for $\lambda / \lambda_{P E}$ dependence on particle size with $\lambda / \lambda_{P E}=1$ at $R \leq R_{c r}$ (see inset 1 to Fig. 9). Note, that in the case $\Delta \mu_{s}>0$ the extrapolation length value decreases. Under the condition when $\Delta \mu_{s}<0$ extrapolation length has to increase resulting into more homogeneous polarization in the nanoparticle.

\section{DISCUSSION}

1. Let us compare our theoretical calculations with experimental dependences $T_{c r}(R)$ and $\bar{\varepsilon}_{i}(R)$ for $\mathrm{BaTiO}_{3}$ and $\mathrm{PbTiO}_{3}$ nanomaterials [15], [16], [3].

Taking into account that $R_{\lambda} \sim 1 / \sqrt{1+2 \varepsilon_{e}}$ (see Eqs. (12), (20),(21a)), i.e. decreases when $\varepsilon_{e}$ increases, and $R_{c}$ saturates when $\varepsilon_{e}$ increases, one can obtain that two cases are 
possible. For ceramics with $\varepsilon_{e}>>1$, one can suppose that $R_{\lambda, c}<<R_{L}$ and use Eqs. (21b), (28c). In contrast, for powder samples $\varepsilon_{e} \sim 1$, one can suppose that $R_{\lambda}>>R_{L}$ and therefore Eqs. (21c) is valid. Really, the estimation of the parameters for aforementioned materials (see, e.g. [17], [11], [18]) have shown that Eqs.(21b) and (21c) can be used for the ceramic and powders description respectively.

Below we regard critical radius as the fitting parameter, so Eqs. (28c) depend on the one fitting parameter $R_{c r}=R_{c r}(T)$ at fixed room temperature $T$. The comparison of calculated on the base of Eqs. (28c) with the experimental $\overline{\varepsilon_{i}}(R)$ dependence for $\mathrm{BaTiO}_{3}$ ceramics is represented in Fig. 10. It is seen good agreement between experimental and calculated data. The difference between obtained critical radii value can be the result of difference in intergrain media due to different technological processes of $\mathrm{BaTiO}_{3}$ ceramics production (see [2], [3] and ref. therein) which can result in difference of surface tension coefficients.

Critical temperature $T_{c r}(R)$ depends on the two parameters: radii $R_{c r}$ and $R_{\lambda}$. The comparison of $(21 \mathrm{c})$ with the experimental dependence $T_{c r}(R)$ for powder $\mathrm{BaTiO}_{3}$ and $\mathrm{PbTiO}_{3}$ samples is represented in Fig. 11. One can see that the theory fits the experimental data rather well.

Using the fitting parameters values, given in the captions to figures 10 and 11, the known values of the electrostriction, elastic stifness, Curie-Weiss constants and critical temperatures (see, e.g. [17], [11]) and the following values of the polarization gradient coefficients for $\mathrm{BaTiO}_{3}$ and $\mathrm{PbTiO}_{3}$ in CGSE-units (see, e.g. [18]): $\delta\left(\mathrm{BaTiO}_{3}\right)=$ $5 \cdot 10^{-15} \mathrm{~cm}^{2}, \quad \delta\left(\mathrm{PbTiO}_{3}\right)=5 \cdot 10^{-16} \mathrm{~cm}^{2}$, one can obtain from Eqs. (15c),(21a), the the following data for physical quantities used in the theory: $\mu_{0}=3.11 \cdot 10^{5} \mathrm{din} / \mathrm{cm}^{2}, \quad \lambda_{P E}=$ $16 \mathrm{~nm}\left(\mathrm{BaTiO}_{3}\right.$ ceramics with $\varepsilon_{e}=100$ and $d=10^{-7}$ CGSE-units); $\mu_{0}=3.28$. $10^{5} \mathrm{din} / \mathrm{cm}^{2}, \quad \lambda_{P E}=50 \mathrm{~nm}\left(\mathrm{BaTiO}_{3}\right.$ powder with $\varepsilon_{e}=1$ and $d=0.55 \cdot 10^{-7} \mathrm{CGSE}$-units $)$. Using the known value of the surface tension of $\mathrm{PbTiO}_{3}$ powder $\mu_{0}=5 \cdot 10^{4} \mathrm{din} / \mathrm{cm}^{2}$ ] and $\varepsilon_{e}=1$ one can obtain $d=1.20 \cdot 10^{-7}$ CGSE units, $\lambda_{P E}=6.9 \mathrm{~nm}$.

One can see that the obtained quantities have reasonable values known for these parameters (see, e.g. 15], 16], 18]). 
2. The surface tension and depolarization field influence were taken into account in the thermodynamic approach to the investigation of ferroelectric nanomaterials properties. Surface energy was expressed via surface tension coefficient that results into extrapolation length dependence on this coefficient. Its value was obtained in the framework of the model taking into account the space-charge layer on the particle surface and shown to be temperature dependent quantity. In the same model the depolarization field was taking into consideration. This field was shown to be partially compensated by the surface charges and proportional to the deviation of the polarization from its mean value. Coefficient of proportionality is defined by the nanoparticle shape and surroundings dielectric permittivity. This lead to the essential difference between dielectric properties of the ceramic and powder samples because of the surrounding media properties difference.

The study of the physical properties of the ferroelectric nanomaterials such as transition temperature, critical particle size, spontaneous polarization and dielectric susceptibility were performed on the base of Euler-Lagrange equation for the polarization with the boundary condition with the temperature dependent extrapolation length of the positive sign. It should be underlined that its negative sign can arise from the negative sign of the coeffient before square polrization gradient. But in this case the higher power gradient terms has to be included into the free energy in order to stabilize the system.

The possibility to calculate physical properties of the nanomaterials by the minimization of the "bulk" free energy density with renormilized coefficients, which depend on the particle size, temperature, surface tension and depolarization field characteristics has been demonstrated. The developed theory gives the basis to the empirical expression proposed in 15] for the $\mathrm{BaTiO}_{3}$ and $\mathrm{PbTiO}_{3}$ transition temperature dependences on the particles size and fits rather well the available experimental data. 


\section{APPENDIX 1}

Let us consider the point charge $q$ located at the distance $r_{0}$ from the center of the dielectric sphere with radius $R>r_{0}$ and dielectric permittivity $\varepsilon_{i}$. The outer space is also regarded as dielectric with permittivity $\varepsilon_{e}$.

In order to find the electric potential $\varphi_{q}^{i, e}$ of this system one have to solve Laplace equation with the appropriate boundary conditions. In this case it has the following form:

$$
\Delta \varphi_{q}^{i, e}=0,\left.\quad \varphi_{q}^{i}\right|_{r=R}=\left.\varphi_{q}^{e}\right|_{r=R},\left.\quad \varepsilon_{i} \nabla \varphi_{q}^{i}\right|_{r=R}=\left.\varepsilon_{e} \nabla \varphi_{q}^{e}\right|_{r=R}
$$

Having solved this equation in the spherical coordinates it is easy to derive the surface contribution to the potential $\varphi_{q}^{i}$ :

$$
\varphi_{q}^{s}\left(r_{0}, r, \theta\right)=q \frac{\varepsilon_{i}-\varepsilon_{e}}{\varepsilon_{i}} \sum_{m=0}^{\infty} \frac{m+1}{m \varepsilon_{i}+(m+1) \varepsilon_{e}} \frac{r_{0}^{m} r^{m}}{R^{2 m+1}} P_{m}(\cos (\theta)) ; \quad r \leq R
$$

where $(r, \theta, \varphi)$ are spherical coordinates and $P_{m}(\cos (\theta))$ is Legendre polynomial of $m$-th order. This potential is determined by the surface bound charges induced by charge $q$. Thus the additional surface energy can be calculated as interaction energy between charge q and induced charges. Considering the electric dipole with the dipole moment $q r_{d}$ directed along polar axes, one can write the interaction energy with respect to $P_{m}(\cos (\theta=0))=1$ as follows

$$
\begin{aligned}
w_{s}\left(r_{0}\right) & =q\left(\left(\varphi_{q}^{s}\left(r_{0}, r_{0}, 0\right)-\varphi_{q}^{s}\left(r_{0}+r_{d}, r_{0}, 0\right)\right)-\left(\varphi_{q}^{s}\left(r_{0}, r_{0}+r_{d}, 0\right)-\varphi_{q}^{s}\left(r_{0}+r_{d}, r_{0}+r_{d}, 0\right)\right)\right) \approx \\
& \approx\left(q r_{d}\right)^{2} \frac{\varepsilon_{i}-\varepsilon_{e}}{\varepsilon_{i}} \sum_{m=0}^{\infty} \frac{m+1}{m \varepsilon_{i}+(m+1) \varepsilon_{e}} \frac{m^{2} r_{0}^{2 m-2}}{R^{2 m+1}}
\end{aligned}
$$

Here we used the strong inequality $r_{d}<<r_{0}$. Then the surface energy can be represented in the form of the equations $(5 a)(5 b)$

$$
\Delta \mu^{s}=n \frac{1}{R^{2}} \int_{0}^{R-a} w_{s}\left(r_{0}\right) r_{0}^{2} d r_{0}
$$

Having performed the integration, one can obtain

$$
\Delta \mu^{s}=n \frac{\left(q r_{d}\right)^{2}}{R^{2}} \frac{\varepsilon_{i}-\varepsilon_{e}}{\varepsilon_{i}} \sum_{m=0}^{\infty} \frac{m+1}{m \varepsilon_{i}+(m+1) \varepsilon_{e}} \frac{m^{2}}{2 m+1}\left(1-\frac{a}{R}\right)^{2 m+1}
$$


The majorant series of the series from (A1-4) is equal to

$$
\frac{1}{2\left(\varepsilon_{i}+\varepsilon_{e}\right)} \sum_{m=0}^{\infty}(m+1) b^{m}\left(1-\frac{a}{R}\right)
$$

Here $b=(1-a / R)^{2}$. Sum of the series (A1-5) can be find in the following way

$$
\sum_{m=0}^{\infty}(m+1) b^{m}=\sum_{m=0}^{\infty} \frac{d\left(b^{m+1}\right)}{d b}=\frac{d}{d b}\left(\sum_{m=0}^{\infty} b^{m+1}\right)=\frac{d}{d b}\left(\frac{b}{1-b}\right)=\frac{1}{(1-b)^{2}}
$$

Using this relation it is easy to find the evident dependence of the series (A1-5) on $a / R$ ratio. Owing to the inequality $a<<R$ we finally get the following expression for the additional surface tension (A1-4)

$$
\Delta \mu^{s}=n \frac{1}{8}\left(\frac{r_{d}}{a} q\right)^{2} \frac{\varepsilon_{i}-\varepsilon_{e}}{\varepsilon_{i}\left(\varepsilon_{i}+\varepsilon_{e}\right)}
$$

\section{APPENDIX 2}

Let us proceed to the integration in the equations (23b). Owing to the boundary condition (15b) integration by parts of the second term in $A_{R}$ leads to the following equation for the coefficient $A_{R}$

$$
\begin{aligned}
A_{R}+a_{0}(W(R))^{2} & =\frac{3}{R^{3}} \int_{0}^{R} r^{2} d r\left(1-\frac{\sinh (r \sqrt{a / \delta})}{M(R) r / R}\right)\left[a\left(1-\frac{\sinh (r \sqrt{a / \delta})}{M(R) r / R}\right)+\right. \\
\left.+\delta\left(\frac{R}{r} \frac{d^{2}}{d r^{2}} \frac{\sinh (r \sqrt{a / \delta})}{M(R)}\right)\right] & =a \frac{3}{R^{3}} \int_{0}^{R} r^{2} d r\left(1-\frac{\sinh (r \sqrt{a / \delta})}{M(R) r / R}\right)
\end{aligned}
$$

Since this integral is equal to $W(R)$ one can get the expression (25) for the coefficient $A_{R}$.

For the sufficiently large particles radius $R>>\sqrt{\delta / a}$ it is easy to substitute $\cosh (x)$ and $\sinh (x)$ for $\exp (x) / 2$ and to rewrite expression for $W(R)$ as follows:

$$
W(R)=1-\frac{3}{\xi(\ell+1)-\ell}
$$

Here

$$
\xi=R \sqrt{a / \delta}, \quad \ell=\lambda \sqrt{\frac{a}{\delta}}
$$


Integration in the expression for $B_{R}$ from the equation (23b) leads to the following coefficient form

$$
\begin{aligned}
\frac{B_{R}}{b}= & 1-\frac{1}{8 \xi^{2} M(R)^{4}}\left(9 \xi^{2}+72 \xi^{2} M(R)^{2}+96 \varphi^{3}(\xi \cosh (\xi)-\sinh (\xi))-\right. \\
& -12 \xi^{2} \cosh (2 \xi)+3 \xi^{2} \cosh (4 \xi)-36 \xi M(R)^{2} \sinh (2 \xi)- \\
& \left.-72 \xi^{2} M(R) \operatorname{Sih}(\xi)+24 \xi^{3} \operatorname{Sih}(2 \xi)+24 \xi^{2} M(R) \operatorname{Sih}(3 \xi)-12 \xi^{3} \operatorname{Sih}(4 \xi)\right)
\end{aligned}
$$

Here and $\operatorname{Sih}(\xi)=\int_{0}^{\xi} d \zeta \sinh (\zeta) / \zeta$ is the integral hyperbolic sine function. When using the strong inequality (21c) in the form $\xi>>1$ and the asymptotic form of $\operatorname{Sih}(\xi) \approx \exp (\xi) /(2 \xi)$ one can suppose that $M(R)=\exp (\xi)(\ell+1) / 2$ and obtain the following expression for $B_{R}$

$$
B_{R} \approx b\left(1-\frac{3}{\xi(\ell+1)^{2}}(4 \ell+1)\right)
$$

It is seen that under the condition

$$
\lambda \sqrt{\frac{a}{\delta}}=\ell>>1
$$

coefficient $B_{R}$ is close to $b$.

Coefficient $C_{R}$ also can be expressed in terms of the hyperbolic functions and the integral hyperbolic sine function, but the final expression is very cumbersome and omitted for the sake of briefness. Using inequalities (21c) and (A2-5) we can suppose that

$$
1-\frac{R}{r} \frac{\sinh (r \sqrt{a / \delta})}{M(R)} \approx 1-\frac{R}{r} \frac{2 \sinh (r \sqrt{a / \delta})}{\exp (\xi)(\ell+1)} \approx 1
$$

Therefore when using the explicit form (23b) of the coefficients $B_{R}$ and $C_{R}$ their approximate expression can be easily simplified to

$$
\begin{aligned}
& B_{R} \approx b \\
& C_{R} \approx c
\end{aligned}
$$

The first of these approximate equalities coincides with (A2-4) owing to strong inequality $(\mathrm{A} 2-4)$ 


\section{REFERENCES}

[1] W. L. Zhong, B. Jiang, P. L. Zhang, J.M. Ma, H. M. Cheng, Z. H. Jang and X. L. Li, J. Phys.:Cond Matter, 5, 2619 (1993).

[2] J. C. Niepce, Electroceramics, 4, Sept. 5-7, 29 (1994).

[3] A. V. Ragulya, V. V. Skorokhod and A. V. Polotai, Poroshkovaya Metallurgiya (Powder Metallurgy), 1/2, 32 (2001).

[4] W. L. Zhong, Y. G. Wang, P. L. Zhang and B. D. Qu, Phys. Rev. B, 50, 698 (1994).

[5] C. L. Wang and S. K. P. Smith, J. Phys.:Cond Matter, 7, 7163 (1993).

[6] R Kretschmer, and K. Binder, Phys. Rev. B, 20, 1065 (1979).

[7] W. Y. Shih, W. Y. Shih and J. A. Aksay, Phys. Rev. B, 50, 15575 (1994).

[8] Wenhui Ma, Mingsheng Zhang and Zuhong Lu, Phys. Stat. Sol. A, 166, 811 (1998).

[9] L. Landau, E. Lifshits, Statistical Physics Part I, Pergamon, Oxford, 1982.

[10] P. Perriat, J. C. Niepce, G. Gaboche, Journal of Thermal Analysis, 41, 635 (1994).

[11] N. A. Pertsev, A. G. Zembilgotov and A. K. Tagantsev, Phys. Rev. Lett., 80, 1988 (1998).

[12] L. Landau, E. Lifshits, Electrodynamics of continuous media, Pergamon, Oxford, 1982.

[13] Y. G. Wang, W. L. Zhong, P. L. Zhang, Sol. State Commun., 90, 329 (1994).

[14] J. Rychetsky and O. Hudak, J. Phys.:Cond Matter, 9, 4955 (1997).

[15] K. Uchino, E. Sadanaga and T. Hirose, J. Am. Ceram. Soc., 721555 (1989).

[16] K. Ishikawa, Phys. Rev. B, 37, 5852 (1988).

[17] F. Jona and G. Shirane, Ferroelctric Crystals, Pergamon, London (1962).

[18] B. D. Qu, W. L. Zhong, and R. H. Prince, Phys. Rev. B, 55, 11218 (1997). 


\section{FIGURE CAPTIONS}

Figure 1. The size dependence of transition temperature at $\theta_{0}=0.005, \varepsilon_{e}=95$ and $\rho_{0}=0.05$ for different $\lambda_{0}$ and $\eta: \lambda_{0}=25$ (curves 1), $\lambda_{0}=250$ (curves 2 ), $\eta=100$ (solid curves), $\eta=0$ (dashed curves), $\varepsilon_{e}=0$ (dotted curves), approximate formulas (dash-dotted

curves), $\mu_{0}=0$ (dash-double-dotted curves). Inset: the same dependences over the inverse radius.

Figure 2. The dependences of the inverse static susceptibility on inverse radius $\left(\chi_{b}\right.$ is bulk static susceptibility) at fixed temperature $\theta_{0}\left(T / T_{C}-1\right)=-0.005$. Inset: the same dependences of $\bar{\chi} / \chi_{b}$ on $R / R_{0}$. The parameters for the different curves correspond to those in Fig.1.

Figure 3. The temperature dependences of inverse and direct (inset) static susceptibility at fixed radius $R / R_{0}=50$. The parameters for the different curves correspond to those in Fig.1.

Figure 4. The size dependence of spontaneous polarization on the inverse and direct (inset) particle radius ( $P_{S b}$ is bulk polarization) at $\theta_{0}\left(T / T_{C}-1\right)=-0.005$. The parameters for the different curves correspond to those in Fig.1.

Figure 5. The temperature dependences of spontaneous polarization over $T / T_{c}\left(P_{S b}^{0}\right.$ is bulk polarization at zero temperature) at fixed radius $R / R_{0}=50$ and $\theta_{0}\left(T / T_{C}-1\right)=-0.005$. The parameters for the different curves correspond to those in Fig.1.

Figure 6. The spatial distribution of the spontaneous polarization and the depolarization field (inset) at fixed temperature $\theta_{0}\left(T / T_{C}-1\right)=-0.005, R / R_{0}=40$. The parameters for the different curves correspond to those in Fig.1.

Figure 7. The temperature dependence of surface tension coefficient at fixed radius $R / R_{0}=50, \theta_{0}=0.005, \lambda_{0}=25$ and $\rho_{0}=0.05, \eta=100$ and different $\varepsilon_{e}$ values: $\varepsilon_{e}=1$ (curves 1), $\varepsilon_{e}=100$ (curves 2), $\varepsilon_{e}=300$ (curves 3 ), $\varepsilon_{e} \rightarrow \infty$ (curves 4 ). Inset: the same dependence for $\lambda_{0}=250$.

Figure 8. The size dependence of surface tension coefficient $\Delta \mu_{F E}$, parameters are the 
same as in basic plot in Fig.7. Inset: the same dependences for $1 / \bar{\chi}$ in FE-phase.

Figure 9. The temperature dependence of extrapolation length (basic plot), parameters are the same as at basic plot in Fig.7. Inset: the size dependence of extrapolation length at fixed temperature $\theta_{0}\left(T / T_{C}-1\right)=-0.005$.

Figure 10. The comparison of experimental (open squares [2], triangles [3]) and theoretical given by Eq. (28c) (solid curves) dependences of the static permittivity (basic plot) and impedance (inset) over particle diameter $D$ for $\mathrm{BaTiO}_{3}$ ceramics. The fitting parameters: $\varepsilon_{B}=1450, D_{c r}=700 \mathrm{~nm}$ (basic plot), $D_{c r}=1500 \mathrm{~nm}$ (inset).

Figure 11. The comparison of experimental (open squares [15], [16]) and theoretical given by Eq. (21c) (solid curves) dependences of transition temperature $T_{C R}$ over particle diameter $D$ for powder $\mathrm{BaTiO}_{3}$ (basic plot) and $\mathrm{PbTiO}_{3}$ (inset) samples. The fitting parameters: $D_{\lambda}=17.4 \mathrm{~nm}, D_{c r}=1000 \mathrm{~nm}\left(\mathrm{BaTiO}_{3}\right) ; D_{\lambda}=8500 \mathrm{~nm}, D_{c r}=12 \mathrm{~nm}$ $\left(\mathrm{PbTiO}_{3}\right)$. 


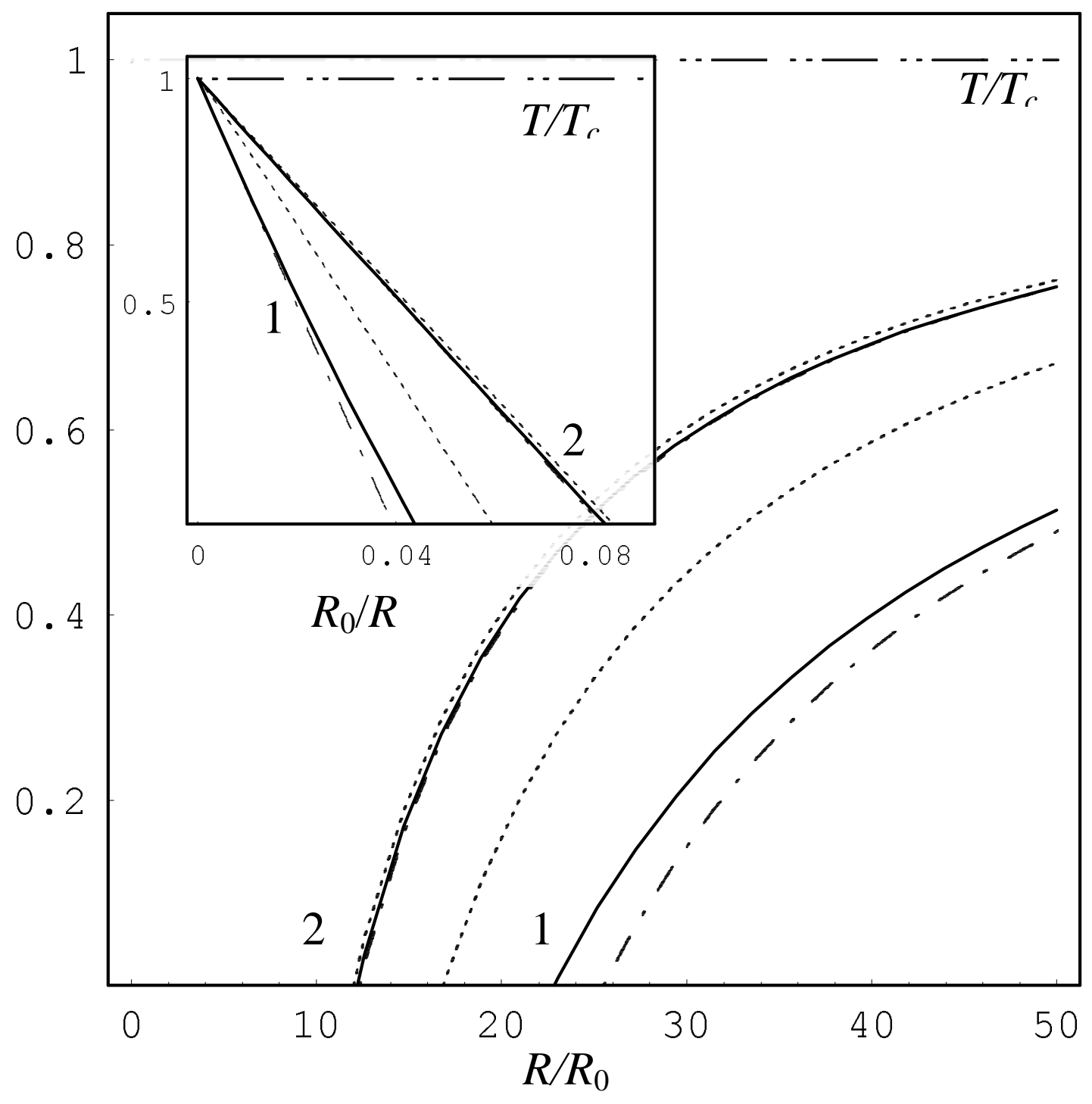




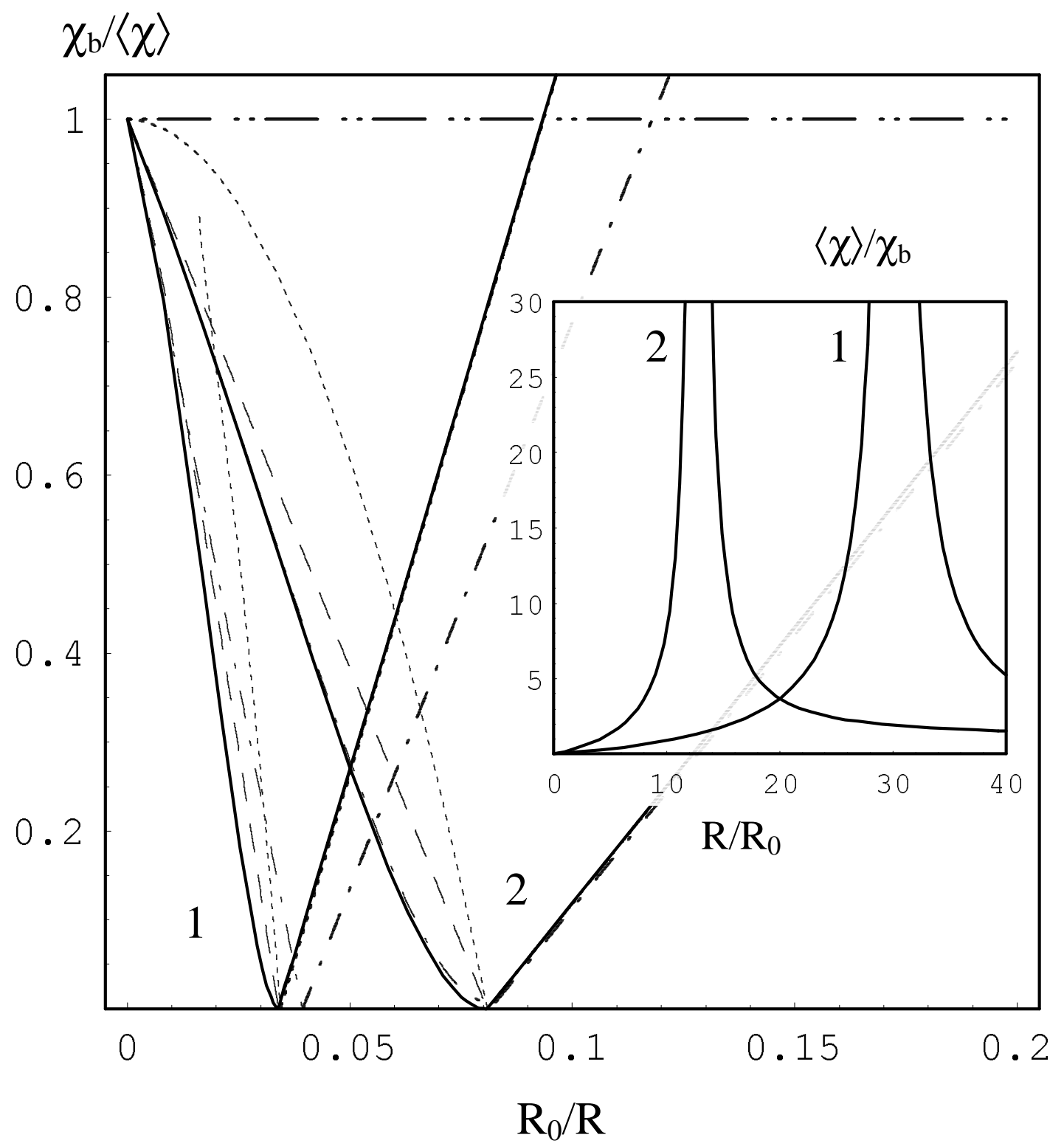




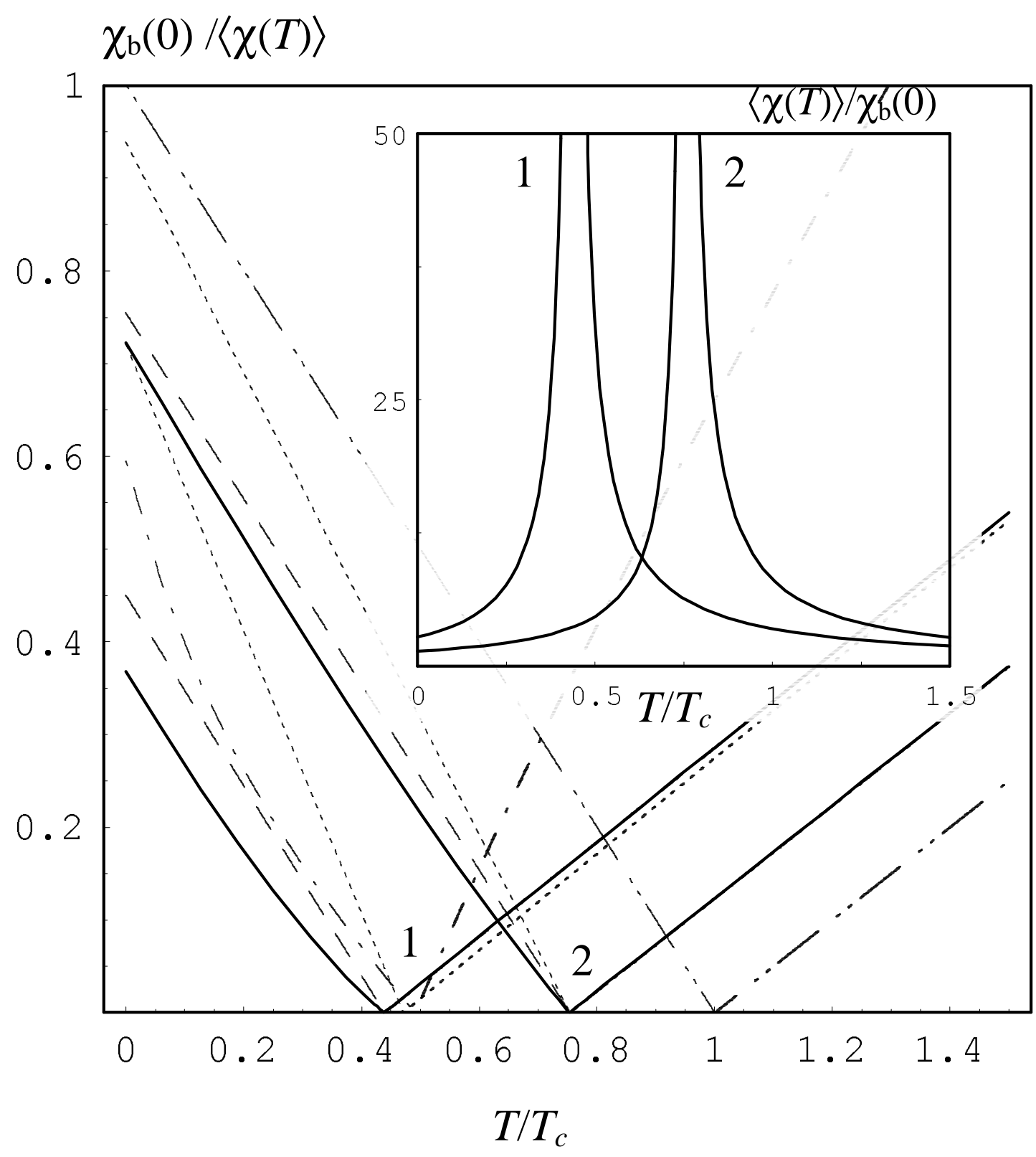




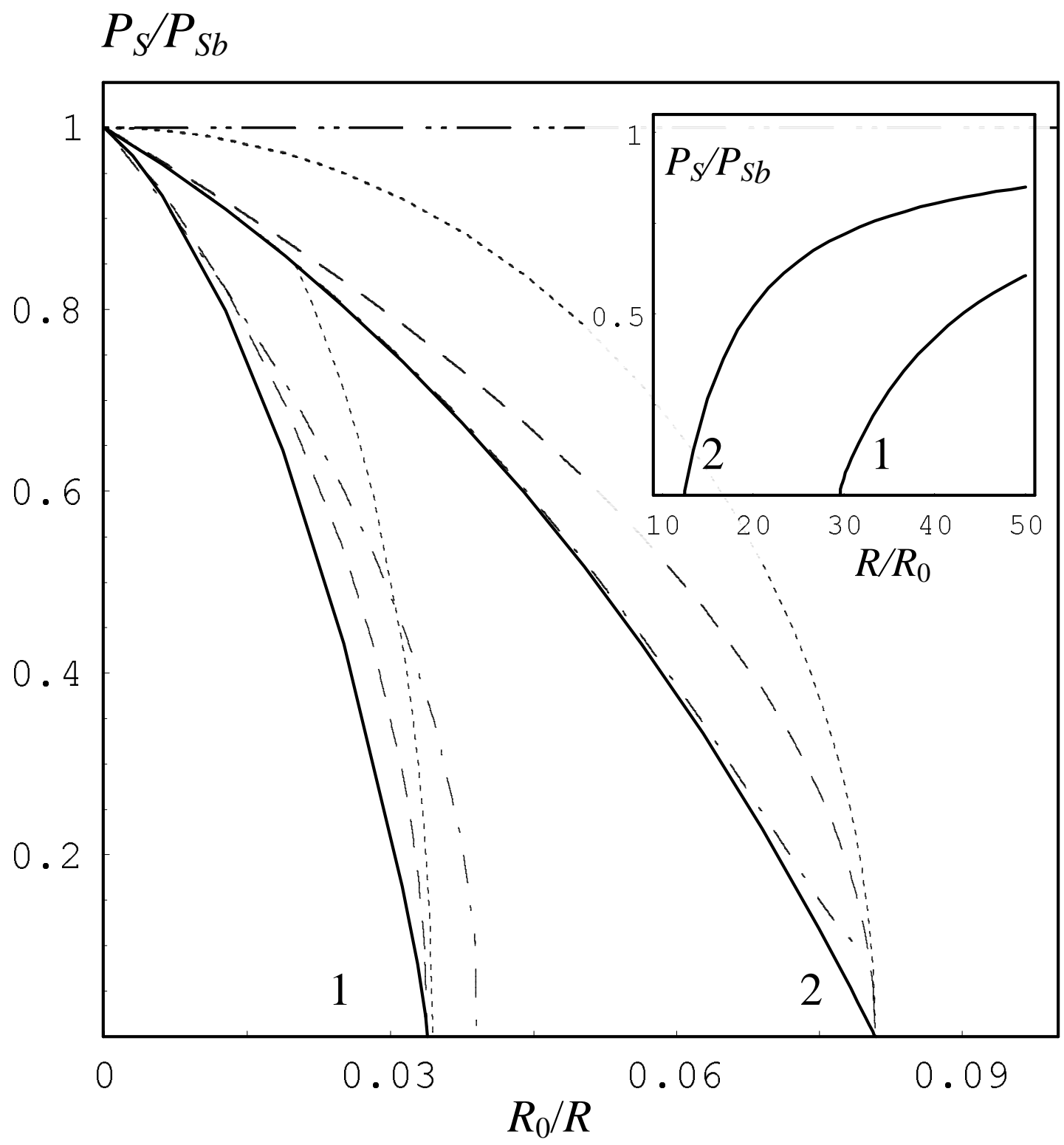




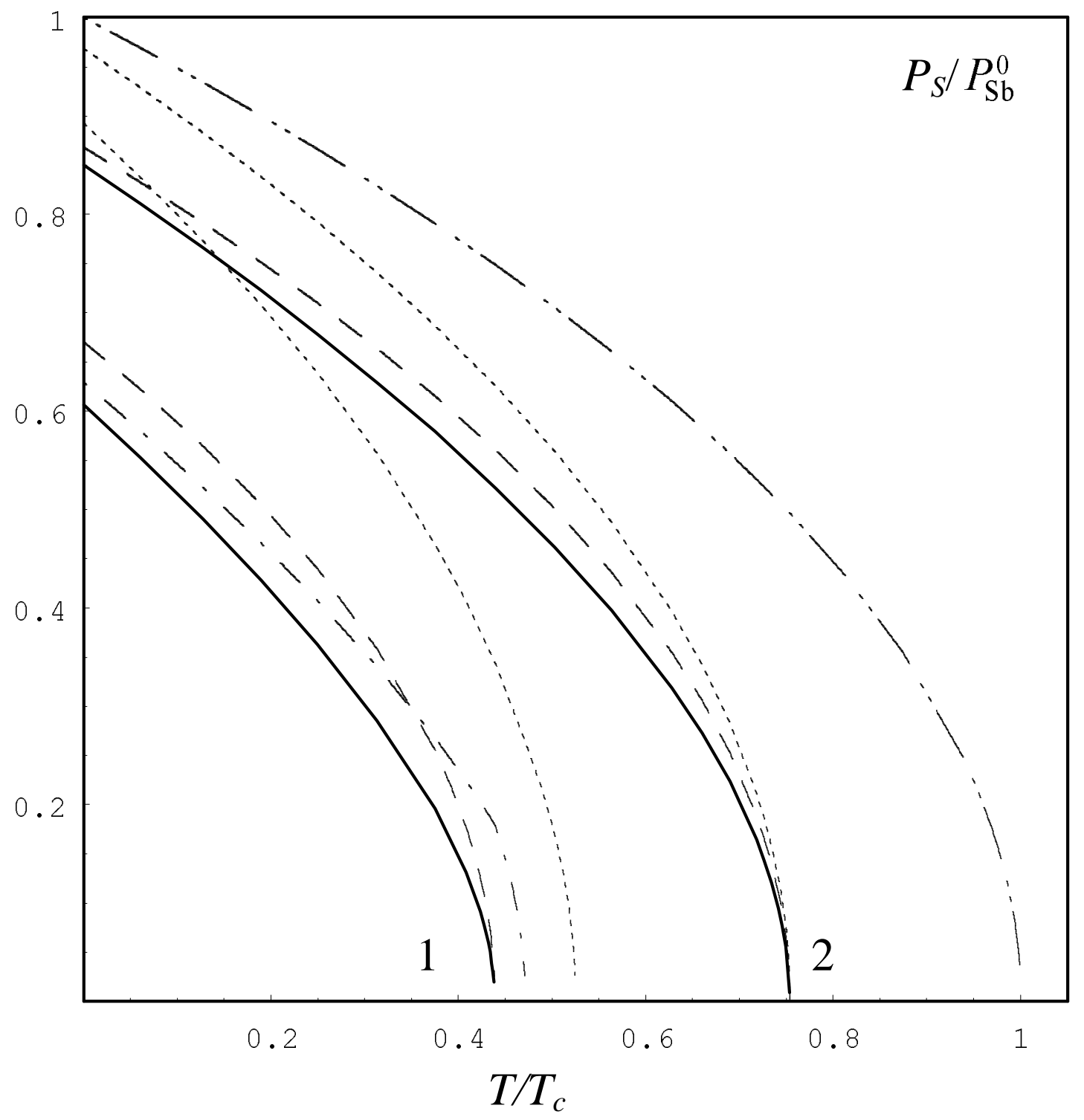




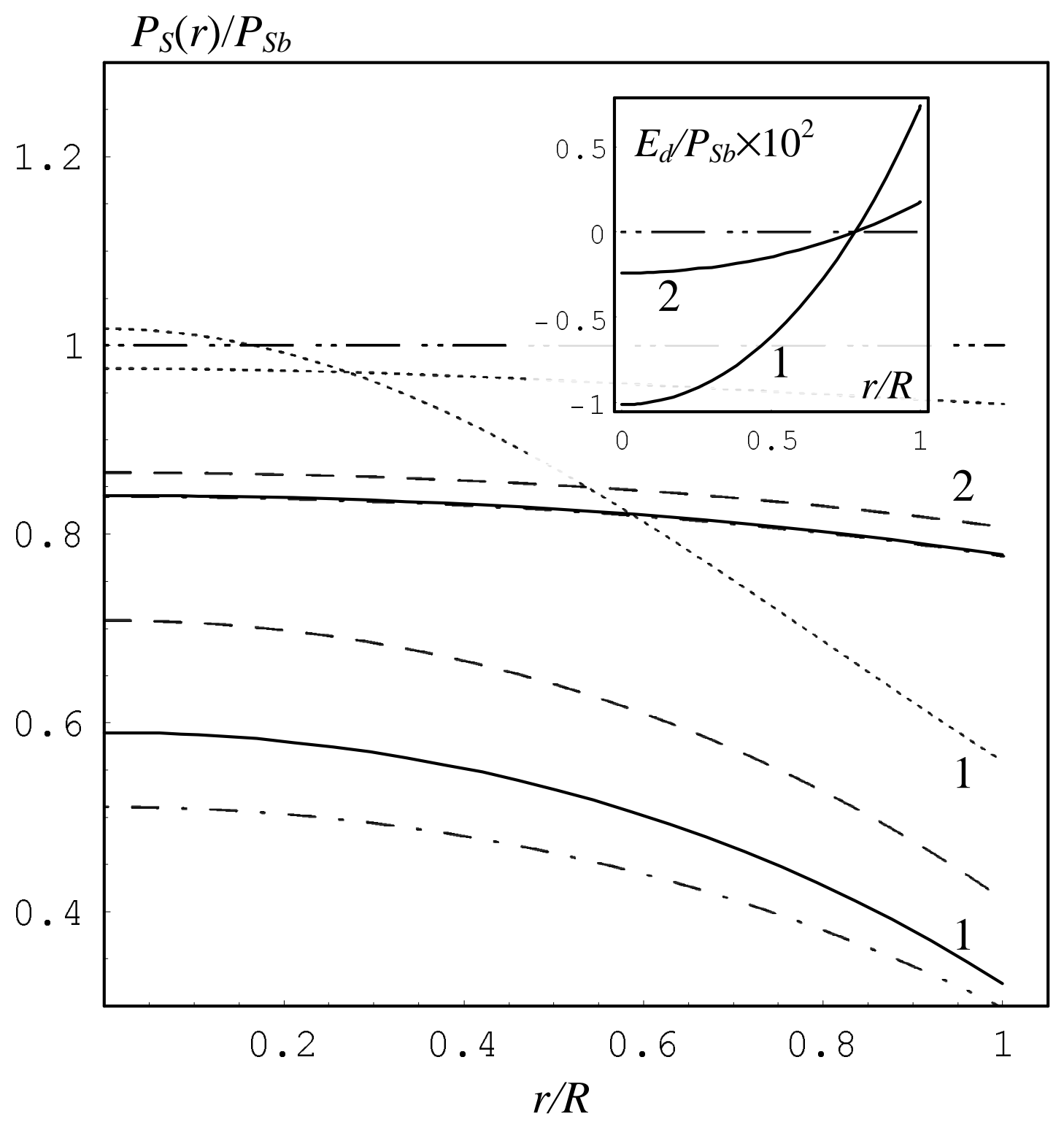




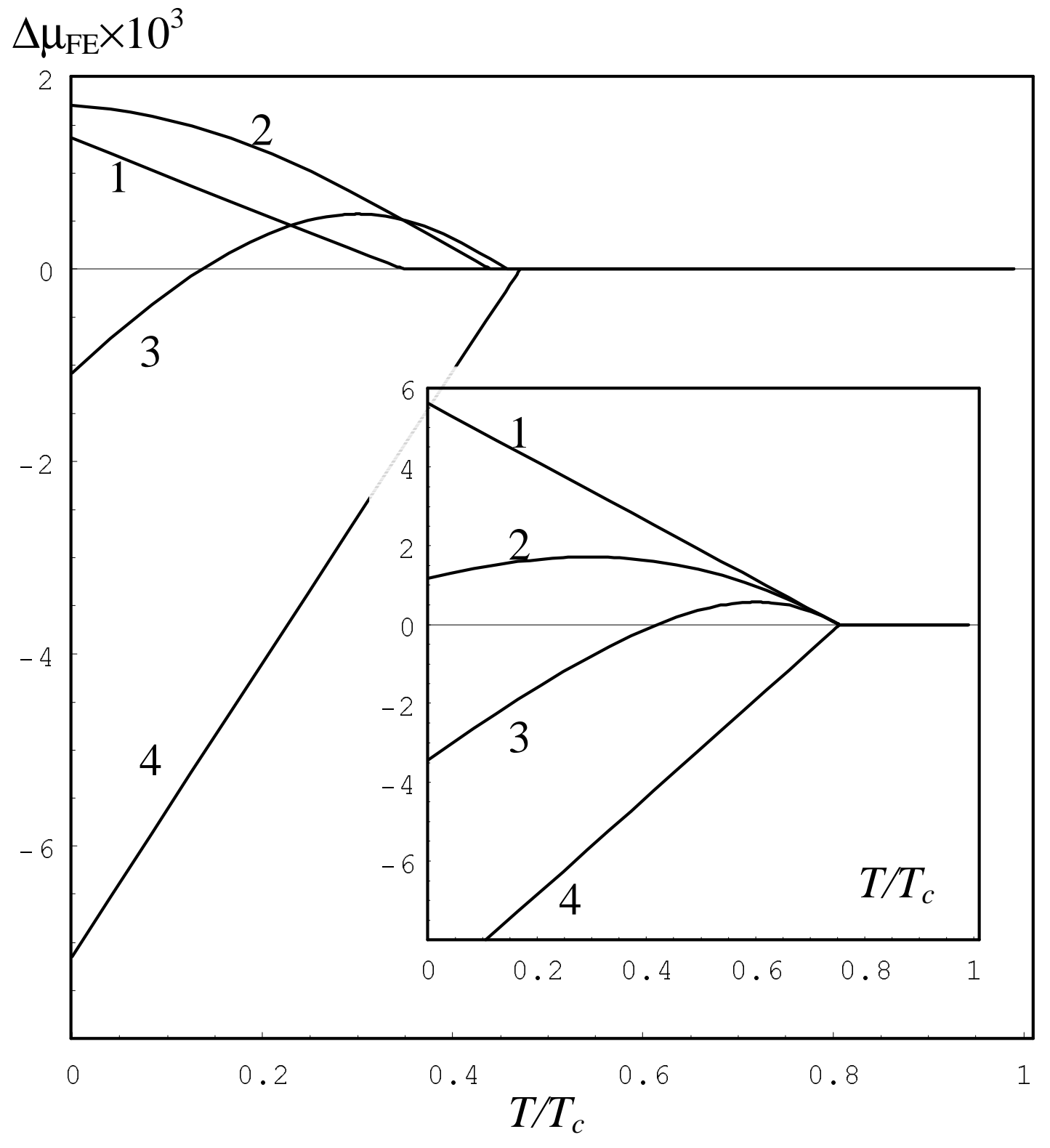




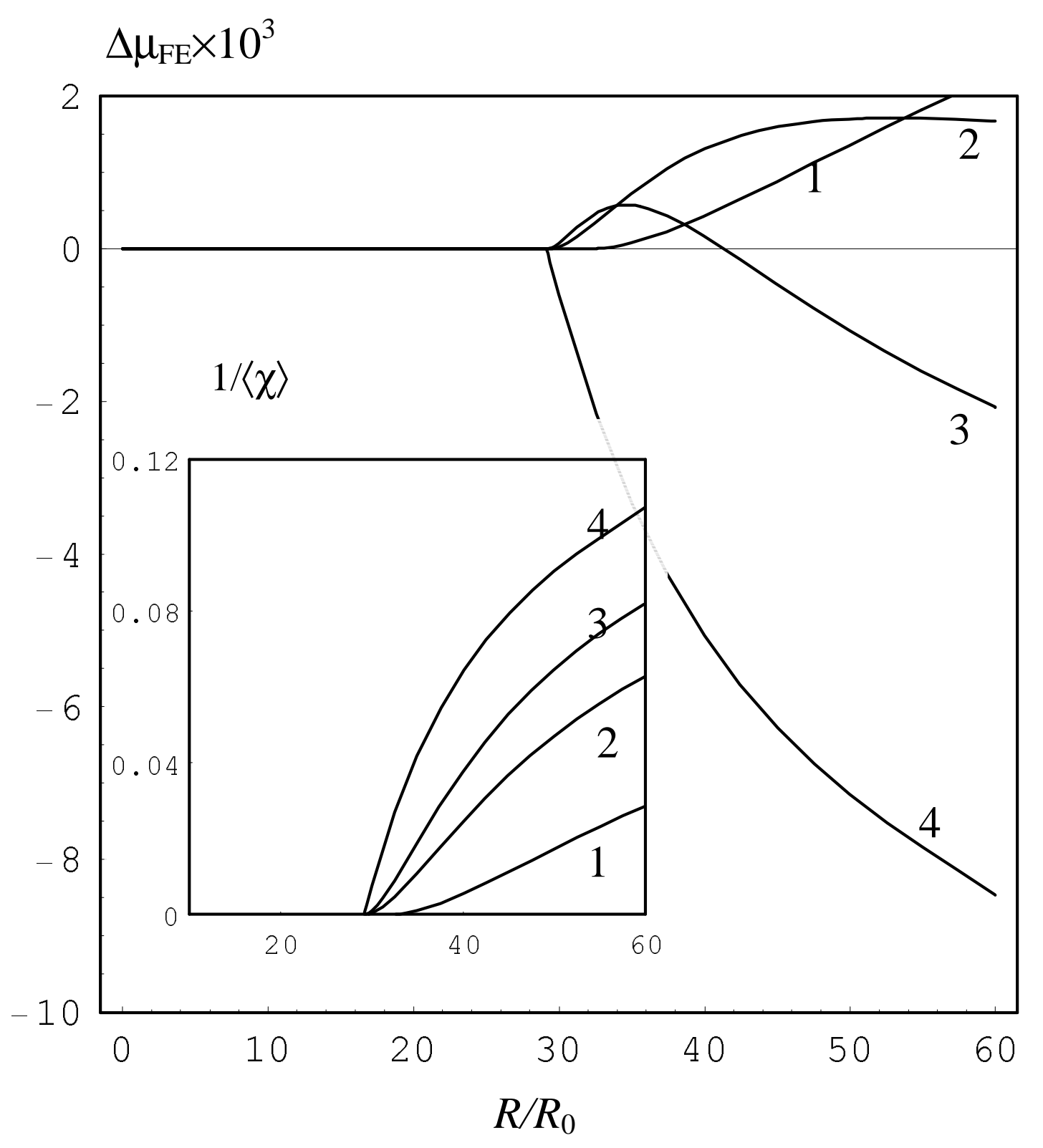




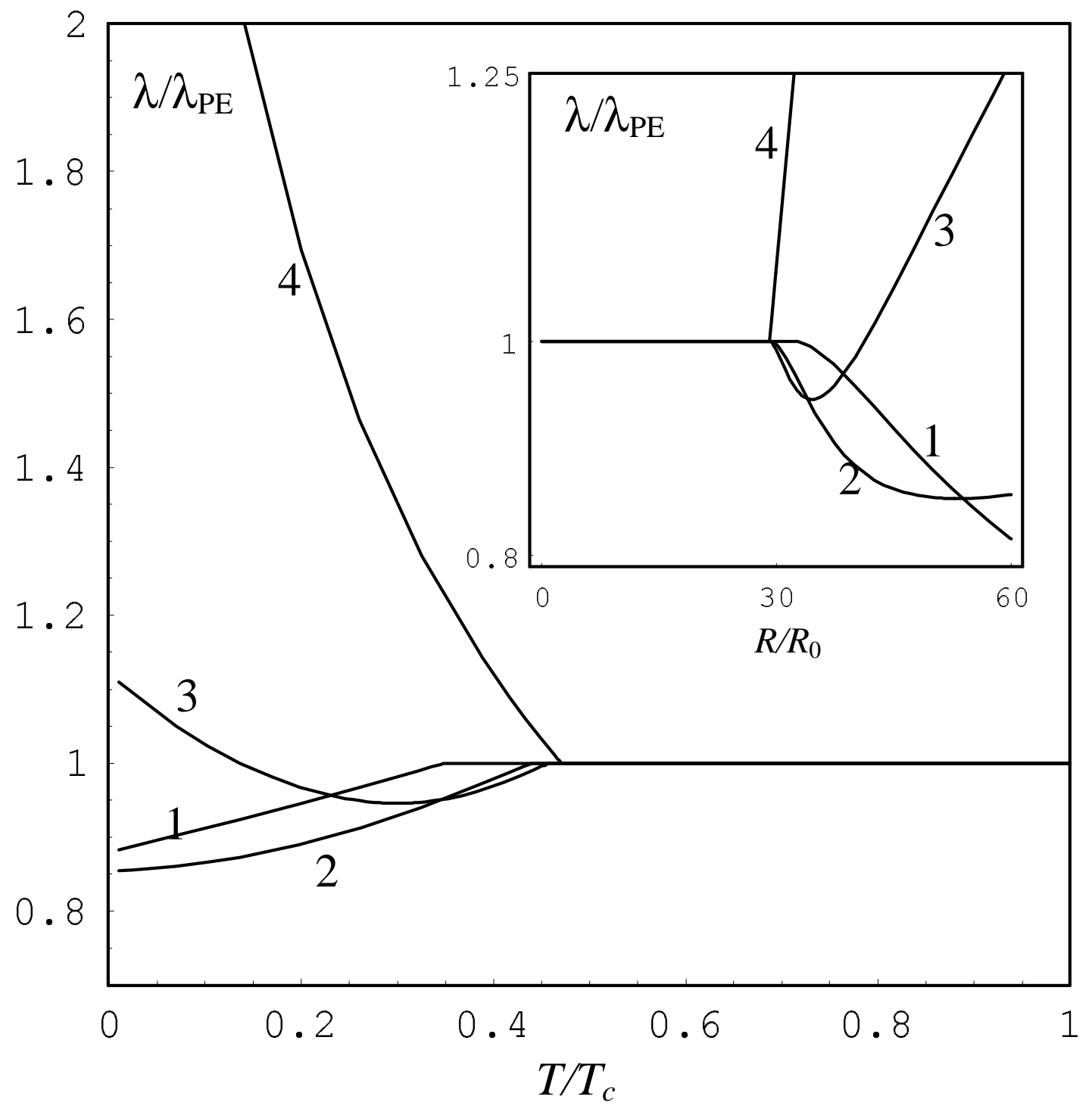




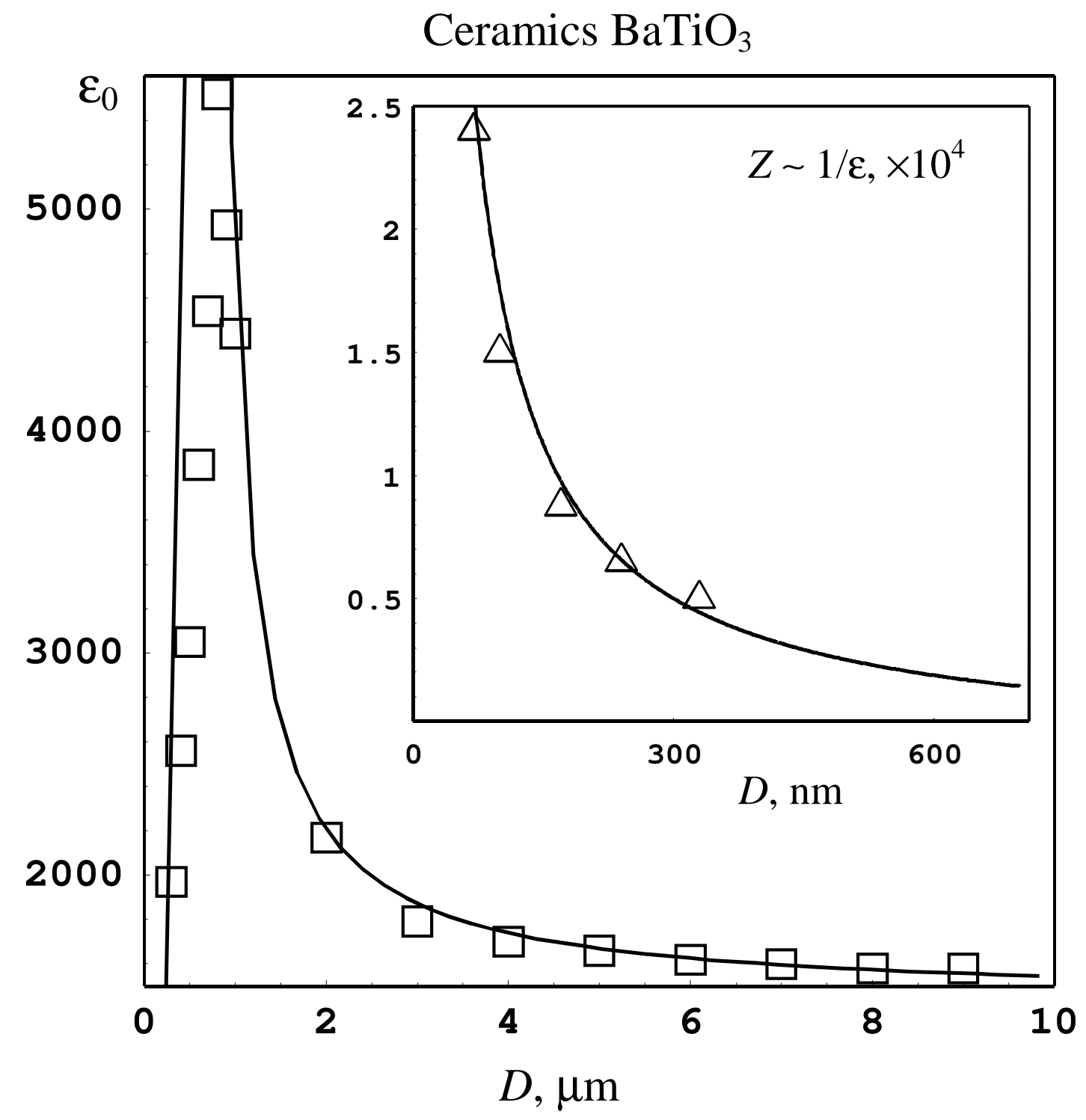




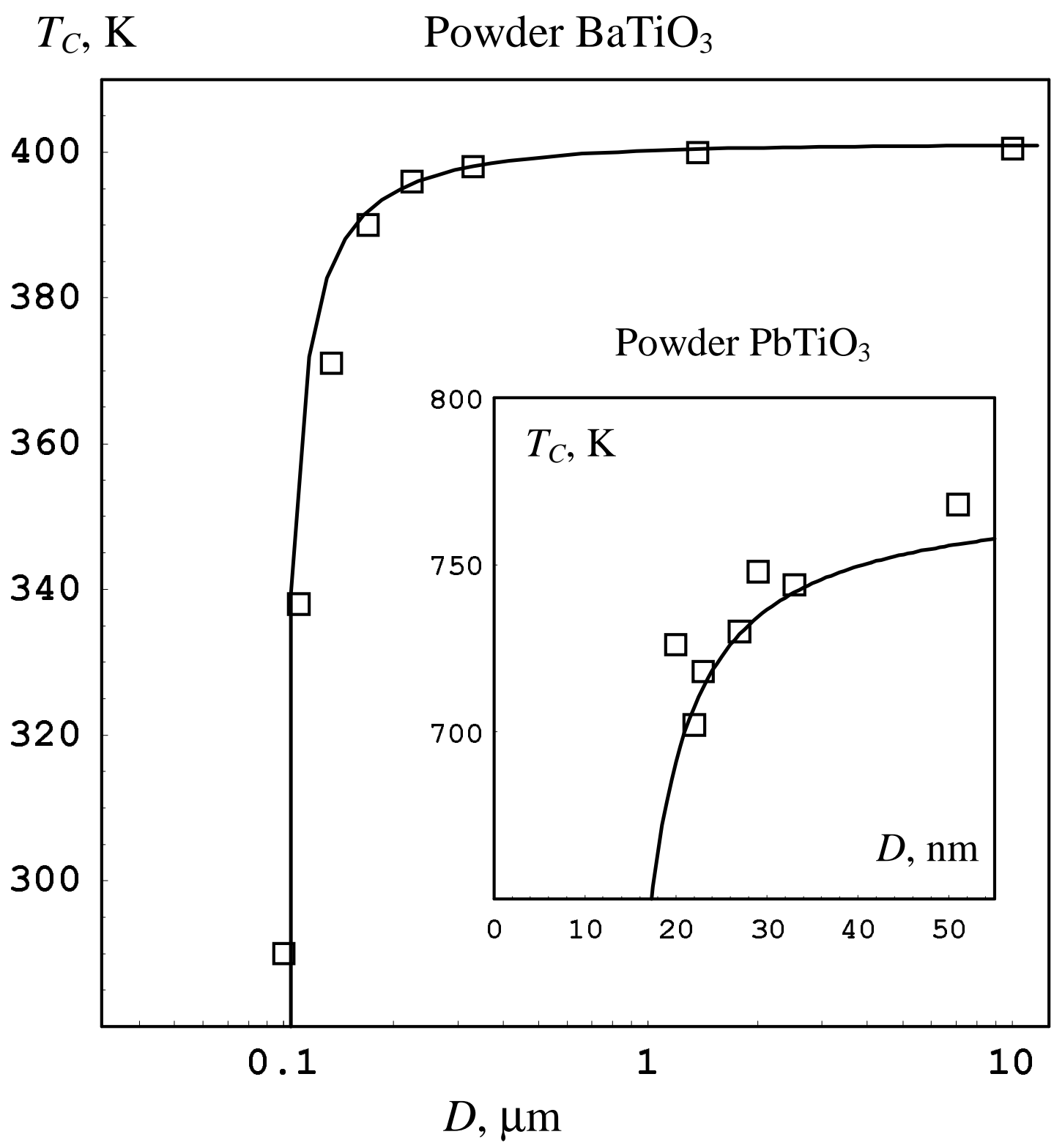

